Article

\title{
Reduced Pre-Lie Algebraic Structures, the Weak and Weakly Deformed Balinsky-Novikov Type Symmetry Algebras and Related Hamiltonian Operators ${ }^{\dagger}$
}

\author{
Orest D. Artemovych ${ }^{1}$, Alexander A. Balinsky ${ }^{2}$, Denis Blackmore ${ }^{3} \mathbb{D}$ and \\ Anatolij K. Prykarpatski ${ }^{4, *}$ \\ 1 Institute of Mathematics, Cracow University of Technology, ul. Warszawska 24, 31155 Cracow, Poland; \\ artemo@usk.pk.edu.pl \\ 2 Mathematics Institute, Cardiff University, Cardiff CF24 4AG, UK; abalinsky@cardif.uk \\ 3 Department of Mathematical Sciences, New Jersey Institute of Technology, University Heights, Newark, \\ NJ 07102, USA; denis.l.blackmore@njit.edu \\ 4 Department of Physics, Mathematics and Computer Sciences, Cracow University of Technology, \\ ul. Warszawska 24, 31155 Cracow, Poland \\ * Correspondence: pryk.anat@ua.fm \\ † Dedicated to Jürgen Moser on his 90th anniversary: A true dynamical systems giant.
}

Received: 29 September 2018; Accepted: 2 November 2018; Published: 6 November 2018

\begin{abstract}
The Lie algebraic scheme for constructing Hamiltonian operators is differential-algebraically recast and an effective approach is devised for classifying the underlying algebraic structures of integrable Hamiltonian systems. Lie-Poisson analysis on the adjoint space to toroidal loop Lie algebras is employed to construct new reduced pre-Lie algebraic structures in which the corresponding Hamiltonian operators exist and generate integrable dynamical systems. It is also shown that the Balinsky-Novikov type algebraic structures, obtained as a Hamiltonicity condition, are derivations on the Lie algebras naturally associated with differential toroidal loop algebras. We study nonassociative and noncommutive algebras and the related Lie-algebraic symmetry structures on the multidimensional torus, generating via the Adler-Kostant-Symes scheme multi-component and multi-dimensional Hamiltonian operators. In the case of multidimensional torus, we have constructed a new weak Balinsky-Novikov type algebra, which is instrumental for describing integrable multidimensional and multicomponent heavenly type equations. We have also studied the current algebra symmetry structures, related with a new weakly deformed Balinsky-Novikov type algebra on the axis, which is instrumental for describing integrable multicomponent dynamical systems on functional manifolds. Moreover, using the non-associative and associative left-symmetric pre-Lie algebra theory of Zelmanov, we also explicate Balinsky-Novikov algebras, including their fermionic version and related multiplicative and Lie structures.
\end{abstract}

Keywords: nonassociative algebra; loop algebra; Lie-Poisson structure; Hamiltonian operator; R-structure; toroidal loop algebra; Poisson structure; Hamiltonian system; derivation; BalinskyNovikov algebra; weak Balinsky-Novikov algebra; weakly deformed Balinsky-Novikov algebra; reduced pre-Lie algebra; fermionic Balinsky-Novikov algebra; Lie algebra; Lie derivation; Leibniz algebra; Riemann algebra

PACS: 02.20.Sv; 02.20.Qs; 02.10.Hh; 02.30.1k; 03.65.Fd; 11.25.-w; 11.25.Hf

MSC: 17B68; 35Q53; 37K10; 58J70; 17D25; 17B20; 17A36 


\section{Introduction}

A left pre-Lie algebra $(A,+, 0)$ is a vector space $A$ over an algebraically closed field $\mathbb{F}$ with a bilinear map $\circ: A \otimes A \rightarrow A$, satisfying the relation

$$
(a \circ b) \circ c-a \circ(b \circ c)=(a \circ c) \circ b-a \circ(c \circ b)
$$

for any $a, b, c \in A$. This is just the invariance of the associator $(a, b, c)=(a \circ c) \circ b-a \circ(c \circ b)$ under the interchange of $b, c \in A$. Hence, every associative algebra is also a pre-Lie algebra, as the associator vanishes identically. It follows from (1) that usual anti-symmetrization yields a Lie bracket

$$
[a, b]:=a \circ b-b \circ a
$$

on $A$ for arbitrary $a, b \in A$. However, not every Lie algebra arises from a pre-Lie algebra. These algebras have been used, under various names, for a long time. As is known [1,2], they were called left-symmetric algebras in the work of Vinberg [3] on convex homogeneous cones, and so were dubbed as Vinberg algebras in some papers. They also appear in the study of affine manifolds, named as right-symmetric algebras [4]. It was proposed in [1] to adopt the name pre-Lie algebras, which had been used by Gerstenhaber [5] as the Lie bracket on the Hochschild cohomology, which arises as a pre-Lie algebra structure on cochains. These pre-Lie algebras have applications in many fields, including perturbative quantum field theory [6,7], where insertion of Feynman graphs into each other equips them with a pre-Lie structure which controls the combinatorics of renormalization.

Moser pointed out the importance of connections between Lie algebraic structures and Hamiltonian dynamics, especially with regard to questions of integrability, in numerous contributions including [8-10]. The fact that many of the integrable Hamiltonian systems discovered during the last several decades have been shown to depend intimately on the Lie-algebraic properties of their internal hidden symmetry structures [11-15] has more than served to confirm Moser's observations. A first account of the Hamiltonian operators and related differential-algebraic relationships, lying in the background of integrable systems and coinciding with reduced pre-algebraic structures, was given by Gelfand and Dorfman [16,17] and later extended by Dubrovin and Novikov [18,19], and also by Balinsky and Novikov [20]. In addition, new special differential-algebraic techniques were devised [21-23] for studying the Lax integrability and the structure of related Hamiltonian operators for a wide class of the Riemann type hydrodynamic hierarchies. Recently, much work [21,24-29] has been devoted to the finite-dimensional representations of the reduced pre-Lie algebraic structures now called the Balinsky-Novikov algebras. Their importance for constructing integrable multi-component nonlinear Camassa-Holm type dynamical systems on functional manifolds was demonstrated by Strachan and Szablikowski [30]. Moreover, they suggested in part the Lie-algebraic embedding of the Balinsky-Novikov algebra in the general Lie-Poisson orbits scheme of classifying Lax integrable Hamiltonian systems. It is worth mentioning the related work [31] by Holm and Ivanov, where integrable multi-component nonlinear Camassa-Holm type dynamical systems were also constructed.

We have devised a formal differential-algebraic recasting of the classical Lie algebraic scheme and developed an effective approach to classification of the underlying algebraic structures of integrable multi-component and multi-dimensional Hamiltonian systems. In particular, we have devised a simple algorithm, based on the Lie-Poisson structure analysis on the adjoint space to toroidal Lie algebras, rigged with non-associated and noncommutative algebras, which enables singling out new algebraic pre-Lie algebraic structures, containing the corresponding Hamiltonian operators, which generate integrable multi-component and multidimensional dynamical systems. The theory of these systems was recently started in [32-42] and developed in [43,44]. In particular, we studied nonassociative and noncommutive algebras over $\mathbb{C}$ and the related Lie-algebraic symmetry structures on the torus $\mathbb{T}^{n}$ for $n \in \mathbb{N}$, generating via the Adler-Kostant-Symes scheme multi-component and multi-dimensional Hamiltonian operators. The latter serve for describing integrable heavenly type equations, whose 
theory has been just recently started in $[30,32,39-41,45-48]$ and advanced in $[12,21,43,49,50]$. In the case of multidimensional torus $\mathbb{T}^{n}$ for $n \in \mathbb{N} \backslash\{1\}$ we have constructed a new weak Balinsky-Novikov type algebra, which is instrumental for describing integrable multidimensional and multicomponent heavenly type equations.

We have also studied the current algebra symmetry structures, related with a new weakly deformed Balinsky-Novikov type algebra on the real axis $\mathbb{R}$, which is instrumental for describing integrable multicomponent dynamical systems [51-54] on functional manifolds. We also show that the well-known Balinsky-Novikov algebraic pre-Lie algebraic structures, obtained in $[17,20]$ as a condition for a matrix differential system to be Hamiltonian and in [55-58] as that on a flat torsion free left-invariant affine connection on affine manifolds, affine structures and convex homogeneous cones, arise as a derivation on the Lie-algebra associated with a differential loop algebra.

Using the theory of Zelmanov [59], we introduce and describe Balinsky-Novikov type algebras in detail, including their fermionic version and related multiplicative and Lie structures.

\section{General Setting}

\subsection{Pre-Lie Algebraic Structures and Related Hamiltonian Operators}

Let $(A,+, \circ)$ be a finite-dimensional algebra (in general noncommutative and associative) over an algebraically closed field $\mathbb{F}$ endowed with a nondegenerate symmetric trace-like $[30,60]$ bilinear form $\langle\cdot, \cdot\rangle: A \otimes A \rightarrow \mathbb{F}$. We shall require that $A$ allows a natural Lie algebra extension $\left(\mathcal{L}_{A},+,[\cdot, \cdot]\right)$ via the usual commutator operation $[\cdot, \cdot]: A \otimes A \rightarrow A$, with respect to which $\langle\cdot, \cdot\rangle$ is ad-invariant; that is,

$$
\langle[a, b], c\rangle+\langle b,[a, c]\rangle=0
$$

for any $a, b, c \in \mathcal{L}_{A}$. Using $A$, one can construct the related toroidal algebra $\widetilde{A}$ of smooth mappings $\mathbb{T}^{n} \rightarrow A$ of the $n$-dimensional torus $\mathbb{T}^{n}, n \in \mathbb{Z}_{+}$, and endow it with the suitably generalized commutator operation $[\cdot, \cdot]: \widetilde{A} \otimes \widetilde{A} \rightarrow \widetilde{A}$ subject to the natural pointwise multiplication operation $\circ: \widetilde{A} \otimes \widetilde{A} \rightarrow$ $\widetilde{A}$. The corresponding loop Lie algebra $\mathcal{L}_{\widetilde{A}}$ will be naturally rigged with a generalized symmetric nondegenerate bilinear ad-invariant form $(\cdot, \cdot): \mathcal{L}_{\widetilde{A}} \otimes \mathcal{L}_{\widetilde{A}} \rightarrow \mathbb{F}$, such that

$$
(a, b):=\int_{\mathbb{T}^{n}}\langle a, b\rangle d x=(b, a)
$$

and

$$
([a, b], c)+(b,[a, c])=0
$$

for any $a, b, c \in \mathcal{L}_{\widetilde{A}}$. The form (4) makes possible the natural identification $\widetilde{A}^{*} \simeq \widetilde{A}$; in particular, for a linear functional $u^{*} \in \widetilde{A}$ we also define its adjoint action on $\widetilde{A}$ as

$$
\left(u^{*} \circ a, b\right):=(a, u \circ b)
$$

for a fixed $u \in \widetilde{A}$ and any $a \in \widetilde{A}$. Now, one can naturally identify the space $\mathcal{L}_{\widetilde{A}}^{*}$, adjoint with respect to the form (4) to $\mathcal{L}_{\widetilde{A}}$, with itself and consider further the space $\mathcal{D}\left(\mathcal{L}_{\widetilde{A}}^{*}\right)$ of smooth scalar functions on $\mathcal{L}_{\widetilde{A}}^{*}$ together with its related Lie-Poisson bracket:

$$
\{f, g\}_{0}:=(u,[\nabla f(u), \nabla g(u)])
$$

for any $f, g \in \mathcal{D}\left(\mathcal{L}_{\widetilde{A}}^{*}\right)$, where the weak gradient map $\nabla: \mathcal{D}\left(\mathcal{L}_{\widetilde{A}}^{*}\right) \rightarrow \mathcal{L}_{\widetilde{A}}$ is defined for any $h \in \mathcal{D}\left(\mathcal{L}_{\widetilde{A}}^{*}\right)$ and all $\xi \in \mathcal{L}_{\widetilde{A}} \simeq \mathcal{L}_{\widetilde{A}}^{*}$ at $u \in \mathcal{L}_{\widetilde{A}} \simeq \mathcal{L}_{\widetilde{A}}^{*}$ as

$$
(\xi, \nabla h(u)):=d h(u+\varepsilon \xi) /\left.d \varepsilon\right|_{\varepsilon=0} .
$$


Owing to its definition $[11,14,61,62]$, bracket (7) satisfies the classical Jacobi condition, so it is a powerful tool for constructing the related Hamiltonian operators on $\mathcal{D}\left(\mathcal{L}_{\widetilde{A}}^{*}\right)$. In particular, we call, following $[17,63]$, a smooth map $\vartheta: \mathcal{L}_{\widetilde{A}}^{*} \rightarrow \operatorname{Hom}\left(\mathcal{L}_{\widetilde{A}} ; \mathcal{L}_{\widetilde{A}}^{*}\right)$ a Hamiltonian operator if the related bracket

$$
\{f, g\}:=(\vartheta(u) \nabla f(u), \nabla g(u))
$$

is determined for any $f, g \in \mathcal{D}\left(\mathcal{L}_{\widetilde{A}}^{*}\right)$ and satisfies the Jacobi identity.

As the canonical Lie-Poisson bracket (7) does not involve essentially the loop Lie algebra structure of $\mathcal{L}_{\widetilde{A}}$, we proceed further to a new Lie algebra structure on $\mathcal{L}_{\widetilde{A}}$ via its central extension. Namely, let $\mathcal{L}_{\widehat{A}}:=\mathcal{L}_{\widetilde{A}} \oplus \mathbb{F}$ denote the centrally extended Lie algebra $\mathcal{L}_{\widetilde{A}}$ endowed with the extended Lie bracket

$$
[(a ; \alpha),(b ; \beta)]:=\left([a, b] ; \omega_{2}(a, b)\right)
$$

for any for any $a, b \in \mathcal{L}_{\widetilde{A}}$ and $\alpha, \beta \in \mathbb{F}$, where the 2-cocycle $\omega_{2}: \mathcal{L}_{\widetilde{A}} \times \mathcal{L}_{\widetilde{A}} \rightarrow \mathbb{F}$ is a skew-symmetric bilinear form and satisfies the Jacobi identity:

$$
\omega_{2}([a, b], c)+\omega_{2}([b, c], a)+\omega_{2}([c, a], b)=0
$$

for any $a, b, c \in \mathcal{L}_{\widetilde{A}}$. It is evident that the existence of nontrivial central extensions on $\mathcal{L}_{\widetilde{A}}$ strongly depends on the underlying structure of the algebra $A$ as presented above. However, there are some algebraic properties that allow us to proceed. Namely, assume that a smooth map $\mathrm{D}_{u}: \mathcal{L}_{\widetilde{A}} \rightarrow \mathcal{L}_{\widetilde{A}}$ defines for a fixed $u \in \mathcal{L}_{\widetilde{A}}$ a weak derivation of $\mathcal{L}_{\widetilde{A}}$, that is

$$
\left(c, \mathrm{D}_{u}[a, b]\right)=\left(c,\left[\mathrm{D}_{u} a, b\right]+\left[a, \mathrm{D}_{u} b\right]\right)
$$

for any $a, b, c \in \mathcal{L}_{\widetilde{A}}$. Then, the following important result holds $[60,64]$.

Proposition 1. Let a smooth map $\mathrm{D}_{u}: \mathcal{L}_{\widetilde{A}} \rightarrow \mathcal{L}_{\widetilde{A}}$ be a skew-symmetric weak derivation of $\mathcal{L}_{\widetilde{A}}$, where $u \in \mathcal{L}_{\widetilde{A}} \simeq \mathcal{L}_{\widetilde{A}}^{*}$. Then,

$$
\omega_{2}(a, b):=\left(a, \mathrm{D}_{u} b\right)
$$

for any $a, b \in \mathcal{L}_{\widetilde{A}}$ and $u \in \mathcal{L}_{\widetilde{A}}^{*} \simeq \mathcal{L}_{\widetilde{A}}$ defines a nontrivial 2-cocycle on $\mathcal{L}_{\widetilde{A}}$.

A proof simply requires verifying (11) and is omitted.

There many ways to construct a priori nontrivial derivations on $\mathcal{L}_{\widetilde{A}}$ such as the following simple consequence of Proposition 1 [16,60,64]:

Theorem 2. Let a nondegenerate skew-symmetric endomorphism $\mathcal{R}: \mathcal{L}_{\widetilde{A}} \rightarrow \mathcal{L}_{\widetilde{A}}$ satisfy the well known Yang-Baxter commutator condition:

$$
[\mathcal{R} a, \mathcal{R} b]=\mathcal{R}([\mathcal{R} a, b]+[a, \mathcal{R} b])
$$

for any $a, b \in \mathcal{L}_{\widetilde{A}}$. Then, the inverse map $\mathcal{R}^{-1}: \mathcal{L}_{\widetilde{A}} \rightarrow \mathcal{L}_{\widetilde{A}}$ is a skew-symmetric derivation of the Lie algebra $\mathcal{L}_{\widetilde{A}}$ and

$$
\omega_{2}(a, b)=\left(a, \mathcal{R}^{-1} b\right)
$$

defines a 2-cocycle on $\mathcal{L}_{\widetilde{A}}$ for any $a, b \in \mathcal{L}_{\widetilde{A}}$.

Remark 3. An interesting consequence of Theorem 2 is that the subspaces

$$
\mathcal{L}_{\widetilde{A}}^{ \pm}:=1 / 2(\mathrm{I} \pm \mathcal{R}) \mathcal{L}_{\widetilde{A}}
$$


are Lie subalgebras of $\mathcal{L}_{\widetilde{A}^{\prime}}$ splitting it into the direct sum

$$
\mathcal{L}_{\widetilde{A}}^{+} \oplus \mathcal{L}_{\widetilde{A}}^{-}=\mathcal{L}_{\widetilde{A}}
$$

In particular, the $\mathcal{R}$-structures on $\mathcal{L}_{\widetilde{A}}$ can be used for constructing additional Hamiltonian operators on $\mathcal{L}_{\widetilde{A}}^{*}$. More precisely, we endow, following [17], $\mathcal{L}_{\widetilde{A}}^{*}$ with the natural differential algebraic structure assuming it to be a polynomial differential algebra $\widetilde{A}(u)$, generated by an element $u \in \widetilde{A}$ and its derivatives $u^{(j)} \in \widetilde{A}\left(j \in \mathbb{Z}_{+}\right)$with respect to be the standard derivation $D_{x}:=\partial / \partial x, x \in \mathbb{S}^{1}$, on $\widetilde{A}$. On $\widetilde{A}(u)$, one can naturally define the space of linear uniform gradient-wise derivations $\Gamma_{\widetilde{A}}(u)$ as

$$
\nabla: \widetilde{A}(u) \rightarrow \operatorname{Der} \widetilde{A}(u),
$$

where $\left[\nabla_{h}, D_{x}\right]=0$ for any $h \in \widetilde{A}(u)$ and the expression

$$
\nabla_{h}:=\sum_{j \in \mathbb{Z}_{+}} h^{(j)}(u) \partial / \partial u^{(j)}
$$

acts on any $f \in \widetilde{A}(u)$ as

$$
\left(\nabla_{h} f\right)(u):=\sum_{j \in \mathbb{Z}_{+}}\left\langle h^{(j)}(u), \partial f(u) / \partial u^{(j)}\right\rangle .
$$

Taking into account the action of the derivations $-\widetilde{A}(u)$ on $\widetilde{A}(u)$, one can rig it with a natural Lie algebra structure

$$
\left[\nabla_{h}, \nabla_{g}\right]:=\nabla_{\{h, g\}}
$$

where the element

$$
\{h, g\}:=g^{\prime}(h)-h^{\prime}(g) \in \widetilde{A}(u)
$$

is written by means of the standard Fréchet derivative on $\widetilde{A}(u)$ :

$$
f^{\prime}(h):=\nabla_{h} f
$$

for any $h, f \in \widetilde{A}(u)$. Following [17], on $\widetilde{A}(u)$, supplemented with a unit element, one can determine a space of functionals $\mathcal{F}_{\widetilde{A}}(u)$ as the set of equivalent elements $f \sim h \in \widetilde{A}(u)$ for which $f-h \sim D_{x} g$ for some element $g \in \widetilde{A}(u)$. Such functionals can be denoted as the integrals

$$
\tilde{f}:=\int_{\mathbb{S}^{1}} f(u) d x \in \mathcal{F}_{\widetilde{A}}(u) .
$$

On $\mathcal{F}_{\widetilde{A}}(u)$, there exists a natural differential $\delta: \mathcal{F}_{\widetilde{A}}(u) \rightarrow \Lambda^{1}(\widetilde{A}(u))$ defined for any $\tilde{f} \in \mathcal{F}_{\widetilde{A}}(u)$ as

$$
\delta \tilde{f}\left(\nabla_{h}\right):=\int_{\mathbb{S}^{1}}\left\langle f^{\prime, *}(u)(1), h\right\rangle d x
$$

where the conjugation mapping " $*$ " is taken with respect to the bilinear form (4) on $\widetilde{A}$ introduced above. Owing to the relationship

$$
f^{\prime, *}(u)(1):=\nabla f(u)
$$

for all $u \in \widetilde{A}$, (21) can be rewritten as

$$
\delta \tilde{f}\left(\nabla_{h}\right)=(\nabla f(u), h) .
$$


Using (22), one can successively determine the whole Grassmann algebra $\Lambda(\widetilde{A}(u))$ of differential forms on $\widetilde{A}(u)$, generated $u \in \widetilde{A}$. In particular, suppose a closed nondegenerate differential 2-form $\omega^{(2)} \in \Lambda^{2}(\widetilde{A}(u)), \delta \omega^{(2)}=0$, is given on $\widetilde{A}(u)$. Then, from [11,61,62],

$$
\{\tilde{f}, \tilde{g}\}_{\omega^{(2)}}:=-\omega^{(2)}\left(\nabla_{\tilde{f}}, \nabla_{\tilde{g}}\right)
$$

where, for any $\tilde{f}, \tilde{g} \in \mathcal{F}_{\widetilde{A}}(u)$, the maps

$$
\delta \tilde{f}(\cdot):=\omega^{(2)}\left(\nabla_{\tilde{f}} \cdot\right)=(\delta u, \nabla \tilde{f}(u)), \delta \tilde{g}(\cdot):=\omega^{(2)}\left(\nabla_{\tilde{g}} \cdot \cdot\right)=(\delta u, \nabla \tilde{g}(u)),
$$

on the algebra $\widetilde{A}(u)$ determine for any $u \in \widetilde{A}^{*} \simeq \widetilde{A}$ the corresponding Hamiltonian operator $\vartheta(u)$ : $\mathcal{L}_{\widetilde{A}} \rightarrow \mathcal{L}_{\widetilde{A}}^{*}$ via the identification

$$
\{\tilde{f}, \tilde{g}\}_{\omega^{(2)}}:=-(\vartheta(u) \nabla \tilde{f}(u), \nabla \tilde{g}(u)) .
$$

Thus, we are led making use of $[60,64,65]$ to the following result.

Proposition 4. Suppose that $\mathcal{L}_{\widetilde{A}}$ allows a skew-symmetric nondegenerate $\mathcal{R}$-structure homomorphism $\mathcal{R}$ : $\mathcal{L}_{\widetilde{A}} \rightarrow \mathcal{L}_{\widetilde{A}}$, satisfying the generalized Yang-Baxter condition

$$
[\mathcal{R} a, \mathcal{R} b]-\mathcal{R}([\mathcal{R} a, b]+[a, \mathcal{R} b])=-\alpha[a, b]
$$

for any $a, b \in \mathcal{L}_{\widetilde{A}}$ and $\alpha \in \mathbb{F}$. Then, differential 2-forms $\omega_{j}^{(2)} \in \Lambda^{2}(\widetilde{A}(u))(j=\overline{1,2})$ on the algebra $\widetilde{A}(u)$ defined as

$$
\omega_{1}^{(2)}\left(\nabla_{\tilde{f}}, \nabla_{\tilde{g}}\right):=\left(\nabla \tilde{f}(u), \mathcal{R}^{-1} \nabla \tilde{g}(u)\right)
$$

and

$$
\omega_{2}^{(2)}\left(\nabla_{\tilde{f}}, \nabla_{\tilde{g}}\right):=(u,[\mathcal{R} \nabla \tilde{f}(u), \mathcal{R} \nabla \tilde{g}(u)])
$$

for any $\tilde{f}, \tilde{g} \in \mathcal{F}_{\widetilde{A}}(u)$ are closed. Moreover, the corresponding Hamiltonian operators, determined from (27) and (28) via the identifications

$$
\omega_{1}^{(2)}\left(\nabla_{\tilde{f}}, \nabla_{\tilde{g}}\right):=\left(\vartheta_{1} \nabla \tilde{f}, \nabla \tilde{g}\right), \quad \omega_{2}^{(2)}\left(\nabla_{\tilde{f}}, \nabla_{\tilde{g}}\right):=\left(\vartheta_{2} \nabla \tilde{f}, \nabla \tilde{g}\right),
$$

are compatible; that is, the sum $\lambda \vartheta_{1}+\mu \vartheta_{2}: \mathcal{L}_{\widetilde{A}} \rightarrow \mathcal{L}_{\widetilde{A}}^{*}$ is also a Hamiltonian operator for arbitrary $\lambda, \mu \in \mathbb{F}$.

Sketch of a proof. As (27) is closed a priori, a proof of the proposition consists in checking the closedness of the 2-forms $\omega_{2}^{(2)} \in \Lambda^{2}(\widetilde{\mathbb{A}}(u))$, which is equivalent to (26). Taking into account (27) and (28) and the representation of $\vartheta_{2}: \mathcal{L}_{\widetilde{\mathbb{A}}} \rightarrow \mathcal{L}_{\widetilde{\mathbb{A}}}^{*}$ as the composition $\vartheta_{2}=\vartheta_{1}^{-1} \vartheta_{0}$, where the Hamiltonian operator $\vartheta_{0}: \mathcal{L}_{\widetilde{\mathbb{A}}} \rightarrow \mathcal{L}_{\widetilde{\mathbb{A}}}^{*}$ is naturally determined from $(7)$ as

$$
(u,[\nabla \tilde{f}(u), \nabla \tilde{g}(u)]):=\left(\vartheta_{0} \nabla \tilde{f}(u), \nabla \tilde{g}(u)\right)
$$

for any $\tilde{f}, \tilde{g} \in \mathcal{F}_{\widetilde{\mathbb{A}}}(u)$. This is equivalent to the compatibility of the Hamiltonian operators $\vartheta_{1}$ and $\vartheta_{2}$ on $\widetilde{\mathbb{A}}(u)[11,13,17,60,64]$. Alternatively, if the parameter $\alpha=0,(27)$ determines a 2-cocycle on $\mathcal{L}_{\widetilde{\mathbb{A}}}$ owing to the fact that the inverse map $\mathcal{R}^{-1}: \mathcal{L}_{\widetilde{\mathbb{A}}} \rightarrow \mathcal{L}_{\widetilde{\mathbb{A}}}$ is a derivation on $\mathcal{L}_{\widetilde{\mathbb{A}}}$; that is,

$$
\mathcal{R}^{-1}[a, b]=\left[\mathcal{R}^{-1} a, b\right]+\left[a, \mathcal{R}^{-1} b\right]
$$

for any $a, b \in \mathcal{L}_{\widetilde{\mathbb{A}}}$, presenting a 2-cocycle on $\mathcal{L}_{\widetilde{\mathbb{A}}}$. Consequently, $\lambda \vartheta_{0}+\mu \vartheta_{1}: \mathcal{L}_{\widetilde{\mathbb{A}}} \rightarrow \mathcal{L}_{\widetilde{\mathbb{A}}}^{*}$ is Hamiltonian for arbitrary $\lambda, \mu \in \mathbb{F}$, which also yields the compatibility of $\vartheta_{1}$ and $\vartheta_{2}$ on $\widetilde{\mathbb{A}}(u)$.

Similarly, one can verify the following $[60,64-66]$ so called "quadratic" compatibility result. 
Proposition 5. Let a skew-symmetric $\mathcal{R}$-structure $\mathcal{R}: \mathcal{L}_{\widetilde{A}} \rightarrow \mathcal{L}_{\widetilde{A}}$ on $\mathcal{L}_{\widetilde{A}}$ satisfy (26). Then,

$$
\{\tilde{f}, \tilde{g}\}_{1}:=(u \circ \nabla \tilde{f}(u), \mathcal{R}(u \circ \nabla \tilde{g}(u)))-(\nabla \tilde{f}(u) \circ u, \mathcal{R}(\nabla \tilde{g}(u) \circ u))
$$

and

$$
\{\tilde{f}, \tilde{g}\}_{2}:=(u,[\mathcal{R} \nabla \tilde{f}(u), \nabla \tilde{g}(u)]+[\nabla \tilde{f}(u), \mathcal{R} \nabla \tilde{g}(u)])
$$

defined for any $\tilde{f}, \tilde{g} \in \mathcal{F}_{\widetilde{A}}(u)$, are Poisson and compatible on $\widetilde{A}(u)$.

\subsection{Lie-Poisson Brackets, Skew-Symmetric Derivations and Balinsky-Novikov Type Algebraic Structures}

Here, we consider for any $u \in \widetilde{A} \simeq \widetilde{A}^{*}$ from $\widetilde{A}$ at $n=1$ a simple skew-symmetric derivation $\mathrm{D}_{u}:=u^{*} \frac{\partial}{\partial x}+\frac{\partial}{\partial x} u: \mathcal{L}_{\widetilde{A}} \rightarrow \mathcal{L}_{\widetilde{A}}$ on $\mathcal{L}_{\widetilde{A}}$ acting as

$$
\begin{gathered}
\left(a, \mathrm{D}_{u} b\right):=\left(a,\left(u^{*} \frac{\partial}{\partial x}+\frac{\partial}{\partial x} u\right) b\right)= \\
=\left(u \circ a, \frac{\partial}{\partial x} b\right)+\left(a, \frac{\partial}{\partial x} u \circ b\right)+\left(a, u \circ \frac{\partial}{\partial x} b\right)
\end{gathered}
$$

for any $a, b \in \mathcal{L}_{\widetilde{A}^{\prime}}$ parameterized by an arbitrary yet fixed $u \in \widetilde{A}^{*}$ and modeling the Hamiltonian operator, analyzed in $[17,20]$ and used in [30]. To verify that (34) is a weak derivation of $\mathcal{L}_{\widetilde{A}}$, it suffices to check that the tri-linear Leibniz type relationship

$$
\left(a, \mathrm{D}_{u}[b, c]\right)=\left(a,\left[\mathrm{D}_{u} b, c\right]+\left[b, \mathrm{D}_{u} c\right]\right)
$$

holds for any $a, b$ and $c \in \mathcal{L}_{\widetilde{A}}$. Following simple calculations, taking into account that $u \in \mathcal{L}_{\widetilde{A}}$ and $\frac{\partial}{\partial x} u \in \mathcal{L}_{\widetilde{A}}$ are functionally independent, one finds that (34) is a skew-symmetric weak derivation of $\mathcal{L}_{\widetilde{A}}$ iff the following algebraic constraints are imposed on $A$ :

$$
l_{[a, b]}=\left[l_{a}, l_{b}\right], \quad\left[r_{a}, r_{b}\right]=0,
$$

where any $a, b \in A l_{a}(b):=a \circ b$ and $r_{a}(b):=b \circ a$ denote, respectively, the left and right shifts on the $A$. The commutator expressions (36) imposed on $A$ coincide with those that determine the well-known Balinsky-Novikov algebra (BNA) by means of

$$
(a \circ b) \circ c=(a \circ c) \circ b
$$

and

$$
(a \circ b) \circ c-(b \circ a) \circ c=a \circ(b \circ c)-b \circ(a \circ c),
$$

which hold for any $a, b, c \in A$, and were derived in a similar context by Gelfand and Dorfman [17] and Balinsky and Novikov in [20]. As already mentioned, the algebra, defined by (36) and (38), is a reduced pre-Lie algebra $A$, which was first introduced in [3,5]. In [29] this algebra was also called $a$ Novikov algebra. In particular, commutative BNA's are associative.

It is worth observing that the linearity of (35) with respect to $u \in \widetilde{A} \simeq \widetilde{A}^{*}$ allows the canonical Lie-Poisson [61,62,65] representation:

$$
\{(u, a),(u, b)\}:=\left(u,[a, b]_{D}\right)=\left(a, \mathrm{D}_{u} b\right)
$$

for any $a, b \in \widetilde{A}$, where

$$
[a, b]_{D}:=a \circ \frac{\partial b}{\partial x}-b \circ \frac{\partial a}{\partial x}
$$

is a new skew-symmetric commutator structure imposed on $\widetilde{A}$. Moreover, since the bracket (39) needs here no symmetry and invariance properties (4) and (5), we simply state that it is Poisson iff the commutator (40) generates a weak Lie algebra structure on $\widetilde{A}$, that is 


$$
\left(u,\left[[a, b]_{D}, c\right]_{D}\right)+\left(u,\left[[b, c]_{D}, a\right]_{D}\right)+\left(u,\left[[c, a]_{D}, b\right]_{D}\right)=0
$$

for any fixed $u \in \widetilde{A}^{*}$ and arbitrary elements $a, b, c \in \widetilde{A}$. It follows from (41) that $A$ coincides with the BNA algebra (38).

Example 6. Having defined the Lie bracket

$$
[a, b]_{D}:=\frac{\partial a}{\partial x} \circ \frac{\partial^{2} b}{\partial x^{2}}-\frac{\partial b}{\partial x} \circ \frac{\partial^{2} a}{\partial x^{2}}
$$

for any $a, b \in \widetilde{A}$, one easily deduces from the weak Jacobi condition (41) the reduced pre-Lie algebra structure:

$$
r_{a b}+r_{a} r_{b}+r_{[a, b]}=0, \quad\left\{l_{a}, l_{b}\right\}:=l_{a} l_{b}+l_{b} l_{a}=0 .
$$

Example 7. In the case of the one-dimensional loop algebra $\widetilde{A}$, a commutator Lie structure, defined for any elements $a, b \in \widetilde{A}$ as

$$
[a, b]_{D}:=a \circ\left(\frac{\partial}{\partial x}\right)^{-1} b-b \circ\left(\frac{\partial}{\partial x}\right)^{-1} a,
$$

where the inverse acting as $\left(\frac{\partial}{\partial x}\right)^{-1}(\ldots):=\frac{1}{2}\left[\int_{0}^{x}(\ldots) d y-\int_{x}^{2 \pi}(\ldots) d y\right]$, generates a weak Lie algebra structure iff the following hold [21] for arbitrary $a, b \in A$ :

$$
r_{a \circ b}=\left[r_{a}, r_{b}\right], \quad r_{\{a, b\}}:=r_{a \circ b}+r_{b \circ a}=0 .
$$

Example 8. It was recently shown in [21] that the spatially one-dimensional skew-symmetric bilinear map

$$
[a, b]_{D}=D_{x}^{-1} b \circ D_{x} a-D_{x}^{-1} a \circ D_{x} b,
$$

imposed on $\widetilde{A}$ for any $a, b \in \widetilde{A}$, generates an adjacent Lie algebra $\mathcal{L}_{\widetilde{A}}$ iff the following Riemann type reduced pre-Lie algebra structure holds for all $a, b \in A$.

$$
\left[r_{a}, r_{b}\right]=0, r_{a \circ b}=l_{a \circ b}, l_{[a, b]}=0 .
$$

Example 9. For the two-dimensional toroidal algebra $\widetilde{A}$, one can define for any $a, b \in \widetilde{A}$ the following new commutator structure

$$
[a, b]_{D}:=\frac{\partial a}{\partial x} \circ \frac{\partial b}{\partial y}-\frac{\partial b}{\partial x} \circ \frac{\partial a}{\partial y}
$$

on $\widetilde{A}$, which generates a weak Lie algebra $\mathcal{L}_{\widetilde{A}}$ iff

$$
\left[r_{a}, r_{b}\right]=0=\left[l_{a}, l_{b}\right]
$$

and

$$
\left[r_{a}, l_{b}\right]=0
$$

hold for any $a, b \in A$.

Remark 10. Note that the similar to (51) Lie commutator structure

$$
[a, b]_{D}:=\frac{\partial a}{\partial x} \circ \frac{\partial^{2} b}{\partial y \partial x}-\frac{\partial b}{\partial x} \circ \frac{\partial^{2} a}{\partial y \partial x}
$$


generates an adjacent Lie algebra $\mathcal{L}_{\tilde{A}}$ iff the following degenerate constraint $l_{a \circ b}=0$ holds for all $a, b \in \widetilde{A}$. Similarly, the Lie-commutator structure

$$
[a, b]_{D}:=\frac{\partial^{2} a}{\partial x \partial y} \circ b-\frac{\partial^{2} b}{\partial x \partial y} \circ a
$$

generates no pre-Lie algebraic structure on the algebra A related to a Hamiltonian operator on $\widetilde{A}^{*}$.

\section{Weak and Weakly Deformed Balinsky-Novikov Type Algebras}

In this section, we study nonassociative and noncommutive algebras over $\mathbb{C}$ and the related Lie-algebraic symmetry structures on the torus $\mathbb{T}^{n}$ for $n \in \mathbb{N}$, generating via the Adler-Kostant-Symes scheme multi-component and multi-dimensional Hamiltonian operators. The latter serve for describing integrable heavenly type equations, whose theory has been just recently started in [30,32,39-41,45-48] and advanced in $[12,21,43,49,50]$. In the case of multidimensional torus $\mathbb{T}^{n}$ for $n \in \mathbb{N} \backslash\{1\}$, we have constructed a new weak Balinsky-Novikov type algebra, which is instrumental for describing integrable multidimensional and multicomponent heavenly type equations.

We have also studied the current algebra symmetry structures, related with a new weakly deformed Balinsky-Novikov type algebra on the real axis $\mathbb{R}$, which is instrumental for describing integrable multicomponent dynamical systems on functional manifolds.

\subsection{A Weak Balinsky-Novikov Type Symmetry Algebra}

Let $(A ;+, \cdot)$ be a finite dimensional nonassociative and, in general, noncommutative algebra over the field $\mathbb{C}$, endowed with a nondegenerate symmetric and invariant bilinear form $\langle\cdot, \cdot\rangle$, that is $\langle a, b\rangle=\langle b, a\rangle$ and $\langle a, b \cdot c\rangle=\langle a \cdot b, c\rangle$ for any $a, b$ and $c \in A$. Let also $\tilde{\mathcal{G}}_{ \pm}:=\widetilde{\operatorname{diff}} f_{ \pm}\left(\mathbb{T}^{n}\right) \otimes A$, $n \in \mathbb{N}$, be $A$-valued loop subalgebras of the algebra $\tilde{\mathcal{G}}:=\tilde{\mathcal{G}}_{+} \oplus \tilde{\mathcal{G}}_{-} \quad$ on the torus $\mathbb{T}^{n}$, holomorphic, respectively, inside $\mathbb{D}_{+}^{1}$ and outside $\mathbb{D}_{-}^{1}$ of the unit disk $\mathbb{D}^{1} \subset \mathbb{C}^{1}$, such that for any $\tilde{a}(\lambda) \in \tilde{\mathcal{G}}_{-}$the value $\tilde{a}(\infty)=0$. The loop algebra $\tilde{\mathcal{G}}$ can be naturally identified with a dense subspace of the dual space $\tilde{\mathcal{G}}^{*}$ through the pairing

$$
(\tilde{l}, \tilde{a}):=\underset{\lambda=\infty}{\operatorname{res}} \int_{\mathbb{T}^{n}}<l(x, \lambda), a(x, \lambda)>d x .
$$

Here, we put, by definition [61,67], a $A$-valued loop vector field $\tilde{a} \in \Gamma\left(\tilde{T}\left(\mathbb{T}^{n}\right) \otimes A\right.$ and a $A$-valued loop differential 1-form $\tilde{l} \in \tilde{\Lambda}^{1}\left(\mathbb{T}^{n}\right) \otimes A$, given as

$$
\tilde{a}=\sum_{j=1}^{n} a^{(j)}(x, \lambda) \partial / \partial x_{j}:=\langle a(x ; \lambda), \partial / \partial x\rangle_{\mathbb{E}^{n}}, \quad \tilde{l}=\sum_{j=1}^{n} l_{j}(x, \lambda) d x_{j}:=\langle l(x ; \lambda), d x\rangle_{\mathbb{E}^{n}}
$$

and introduced, for brevity, the gradient operator $\partial / \partial x:=\left(\partial / \partial x_{1}, \partial / \partial x_{2}, \ldots, \partial / \partial x_{n}\right)^{\top}$ and the standard bilinear form $\langle\cdot, \cdot\rangle_{\mathbb{E}^{n}}$ in the Euclidean space $\mathbb{E}^{n}$. The algebra $\tilde{\mathcal{G}}$ can be further equipped with the Lie bracket

$$
[\tilde{a}, \tilde{b}]_{D}=\left\langle\langle a(x ; \lambda), \partial / \partial x\rangle_{\mathbb{E}^{n}} \cdot b(x ; \lambda), \partial / \partial x\right\rangle_{\mathbb{E}^{n}}-\left\langle\langle b(x ; \lambda), \partial / \partial x\rangle_{\mathbb{E}^{n}} \cdot a(x ; \lambda), \partial / \partial x\right\rangle_{\mathbb{E}^{n}} .
$$

Let us assume now that this way obtained $A$-valued vector field algebra $\tilde{\mathcal{G}}$ is a weak Lie algebra $\mathcal{L}_{\tilde{\mathcal{G}}}$, that is

$$
\left(\tilde{l},\left[[\tilde{a}, \tilde{b}]_{D}, \tilde{c}\right]_{D}+\left[[\tilde{b}, \tilde{c}]_{D}, \tilde{a}\right]_{D}+\left[[\tilde{c}, \tilde{a}]_{D}, \tilde{a}\right]_{D}\right)=0
$$

for any $\tilde{a}, \tilde{b}$ and $\tilde{c} \in \mathcal{L}_{\tilde{\mathcal{G}}} \simeq \Gamma\left(\tilde{T}\left(\mathbb{T}^{n}\right) \otimes A\right.$ and arbitrary $\tilde{l} \in \mathcal{L}_{\tilde{\mathcal{G}}}^{*} \simeq \tilde{\Lambda}^{1}\left(\mathbb{T}^{n}\right) \otimes A$. As a result of easy enough 
calculations for the case of the $A$-valued loop vector field Lie algebra $\mathcal{L}_{\tilde{\mathcal{G}}}$ on the torus $\mathbb{T}^{n}$ of dimension $n \in \mathbb{N} \backslash\{1\}$, one finds that the algebra $A$ should satisfy the following algebraic constraints:

$$
R_{a \circ b}=R_{a} R_{b}, \quad L_{a} L_{b}=L_{b} L_{a}
$$

for any $a, b \in A$, where, by definition, $R_{a} b:=b \cdot a$ is the right shift and $L_{a} b:=a \cdot b$ is the left shift on the algebra $A$. This, in particular, means that the canonical $[11,13,14,61,65]$ Lie-Poisson bracket

$$
\{(\tilde{l}, \tilde{a}),(\tilde{l}, \tilde{b})\}:=\left(\tilde{l},[\tilde{a}, \tilde{b}]_{D}\right)
$$

for any $\tilde{a}, \tilde{b} \in \mathcal{L}_{\tilde{\mathcal{G}}}$ and arbitrary element $\tilde{l} \in \mathcal{L}_{\tilde{\mathcal{G}}}^{*}$ satisfies the Jacobi identity (55).

Remark 11. In case of the A-valued loop vector field Lie algebra $\mathcal{L}_{\tilde{\mathcal{G}}} \simeq \Gamma\left(\tilde{T}\left(\mathbb{S}^{1}\right) \otimes A\right.$ on the one-dimensional circle $\mathbb{S}^{1}$, the corresponding algebraic constraints reduce to the following less strong dual Balinsky-Novikov algebra A expressions:

$$
R_{a \circ b}-R_{a} R_{b}=R_{b \circ a}-R_{b} R_{a}, \quad L_{a} L_{b}=L_{b} L_{a}
$$

for any $a, b \in A$. Thereby, the obtained algebra $A$ will be naturally called a weak Balinsky-Novikov type algebra.

Summarizing the reasonings above, we can now formulate the obtained above result as the following theorem.

Theorem 12. The canonical Lie-Poisson bracket (57) on the co-adjoint space $\tilde{\mathcal{G}}^{*} \simeq \tilde{\Lambda}^{1}\left(\mathbb{T}^{n}\right) \otimes A$ in the case $n \in \mathbb{N} \backslash\{1\}$ is compatible with the internal algebraic structure of the algebra $A$ iff it satisfies the weak Balinsky-Novikov algebraic constraints (56).

Observe now that, owing to pairing (53), the corresponding dual spaces $\tilde{\mathcal{G}}_{+}^{*}$ and $\tilde{\mathcal{G}}_{-}^{*}$ satisfy the relationships

$$
\tilde{\mathcal{G}}_{+}^{*} \simeq \tilde{\mathcal{G}}_{-}, \quad \tilde{\mathcal{G}}_{-}^{*} \simeq \tilde{\mathcal{G}}_{+},
$$

where, for any $\tilde{l}(\lambda) \in \tilde{\mathcal{G}}_{-}^{*}$, one can impose the dual constraint $\tilde{l}(0)=0$. Having defined the projections

$$
P_{ \pm}: \tilde{\mathcal{G}} \rightarrow \tilde{\mathcal{G}}_{ \pm} \subset \tilde{\mathcal{G}}
$$

one can construct a classical $\mathcal{R}$-structure $[14,60,65]$ on the Lie algebra $\tilde{\mathcal{G}}$ as the endomorphism $\mathcal{R}$ : $\tilde{\mathcal{G}} \rightarrow \tilde{\mathcal{G}}$, where

$$
\mathcal{R}:=\left(P_{+}-P_{-}\right) / 2,
$$

which allows for determining on the vector space $\tilde{\mathcal{G}}$, the new Lie algebra structure

$$
[\tilde{a}, \tilde{b}]_{\mathcal{R}}:=[\mathcal{R} \tilde{a}, \tilde{b}]+[\tilde{a}, \mathcal{R} \tilde{b}]
$$

for any $\tilde{a}, \tilde{b} \in \tilde{\mathcal{G}}$, satisfying the standard Jacobi identity.

Let $\mathrm{D}\left(\tilde{\mathcal{G}}^{*}\right)$ denote the space of smooth functions on $\tilde{\mathcal{G}}^{*}$. Then, for any $f, g \in \mathrm{D}\left(\tilde{\mathcal{G}}^{*}\right)$, one can write the general canonical $[11,14,65,68]$ Lie-Poisson bracket

$$
\{f, g\}:=(\tilde{l},[\nabla f(\tilde{l}), \nabla g(\tilde{l})]),
$$

where $\tilde{l} \in \tilde{\mathcal{G}}^{*}$ is a seed element and $\nabla f, \nabla g \in \tilde{\mathcal{G}}$ are the standard functional gradients at $\tilde{l} \in \tilde{\mathcal{G}}^{*}$ with respect to the metric (53). The related (62) space of Casimir invariants is defined as the set $I\left(\tilde{\mathcal{G}}^{*}\right) \subset \mathrm{D}\left(\tilde{\mathcal{G}}^{*}\right)$ of smooth independent functions $h_{j} \in \mathrm{D}\left(\tilde{\mathcal{G}}^{*}\right), j=\overline{1, n}$, for which

$$
a d_{\nabla h_{j}(\tilde{l})}^{*} \tilde{l}=0,
$$


where, for any $A$-valued seed element

$$
\tilde{l}=<l, d x>_{\mathbb{E}^{n}}
$$

the gradients

$$
\nabla h_{j}(\tilde{l}):=\left\langle\nabla h_{j}(l), \partial / \partial x\right\rangle_{\mathbb{E}^{n}}
$$

and the coadjoint action (63) can be equivalently rewritten, for instance, as

$$
\left\langle\partial / \partial x,\left(l \nabla h_{j}(l)\right)\right\rangle_{\mathbb{E}^{n}}+\left\langle\partial / \partial x, \nabla h_{j}(l)\right\rangle_{\mathbb{E}^{n}} l=0
$$

for any $j=\overline{1, n}$. If taking two smooth functions $h^{(y)}, h^{(t)} \in I\left(\tilde{\mathcal{G}}^{*}\right) \subset \mathrm{D}\left(\tilde{\mathcal{G}}^{*}\right)$, their second Poisson bracket

$$
\left\{h^{(y)}, h^{(t)}\right\}_{\mathcal{R}}:=\left(\tilde{l},\left[\nabla h^{(y)}, \nabla h^{(t)}\right]_{\mathcal{R}}\right)
$$

on the space $\tilde{\mathcal{G}}^{*}$ vanishes, that is

$$
\left\{h^{(y)}, h^{(t)}\right\}_{\mathcal{R}}=0
$$

at any seed element $\tilde{l} \in \tilde{\mathcal{G}}^{*}$. Since the functions $h^{(y)}, h^{(t)} \in I\left(\tilde{\mathcal{G}}^{*}\right)$, the following coadjoint action relationships hold:

$$
a d_{\nabla h(y)(\tilde{l})}^{*} \tilde{l}=0, \quad a d_{\nabla h^{(t)}(\tilde{l})}^{*} \tilde{l}=0,
$$

which can be equivalently rewritten as

$$
\left\langle\partial / \partial x,\left(l \nabla h^{(y)}(l)\right)\right\rangle_{\mathbb{E}^{n}}+\left\langle\partial / \partial x, \nabla h^{(y)}(l)\right\rangle_{\mathbb{E}^{n}} l=0
$$

and similarly

$$
\left\langle\partial / \partial x,\left(l \nabla h^{(t)}(l)\right)\right\rangle_{\mathbb{E}^{n}}+\left\langle\partial / \partial x, \nabla h^{(t)}(l)\right\rangle_{\mathbb{E}^{n}} l=0 .
$$

Consider now the following Hamiltonian flows on the space $\tilde{\mathcal{G}}^{*}$ :

$$
\begin{aligned}
& \partial \tilde{l} / \partial y:=\left\{h^{(y)}, \tilde{l}\right\}_{\mathcal{R}}=-a d_{\nabla h(y)}^{*}(\tilde{l})_{+} \\
& \tilde{l}, \\
& \partial \tilde{l} / \partial t:=\left\{h^{(t)}, \tilde{l}\right\}_{\mathcal{R}}=-a d_{\nabla h^{(t)}(\tilde{l})_{+}}^{*} \tilde{l},
\end{aligned}
$$

where $h^{(y)}, h^{(t)} \in I\left(\tilde{\mathcal{G}}^{*}\right)$ and $y, t \in \mathbb{R}$ are the corresponding evolution parameters. Since $h^{(y)}, h^{(t)} \in$ $I\left(\tilde{\mathcal{G}}^{*}\right)$ are Casimirs, the flows (72) commute. Thus, taking into account the representations (70), one can recast the flows (72) as

$$
\begin{aligned}
& \partial l / \partial y=-\left\langle\partial / \partial x,\left(l \nabla h^{(y)}(\tilde{l})_{+}\right)\right\rangle_{\mathbb{E}^{n}}+\left\langle\partial / \partial x, \nabla h^{(y)}(\tilde{l})_{+}\right\rangle_{\mathbb{E}^{n}} l, \\
& \partial l / \partial t=-\left\langle\partial / \partial x,\left(l \nabla h^{(t)}(\tilde{l})_{+}\right)\right\rangle_{\mathbb{E}^{n}}+\left\langle\partial / \partial x, \nabla h^{(t)}(\tilde{l})_{+}\right\rangle_{\mathbb{E}^{n}} l .
\end{aligned}
$$

Lemma 13. The compatibility of commuting flows (73) is equivalent to the Lax type vector fields relationship

$$
\frac{\partial}{\partial y} \nabla h^{(t)}(\tilde{l})_{+}-\frac{\partial}{\partial t} \nabla h^{(y)}(\tilde{l})_{+}+\left[\nabla h^{(y)}(\tilde{l})_{+}, \nabla h^{(t)}(\tilde{l})_{+}\right]=0,
$$

which holds for all $y, t \in \mathbb{R}$ and arbitrary $\lambda \in \mathbb{C}$. 
Proof. The compatibility of commuting flows (73) implies that $\partial^{2} l / \partial t \partial y-\partial^{2} l / \partial y \partial t=0$ for all $y, t \in \mathbb{R}$ and arbitrary $\lambda \in \mathbb{C}$. Taking into account the expressions (72), one has for any $A$-valued vector field $\tilde{Z}=<Z, \frac{\partial}{\partial x}>\in \tilde{\mathcal{G}}$

$$
\begin{aligned}
& 0=\left(\partial^{2} \tilde{l} / \partial t \partial y-\partial^{2} \tilde{l} / \partial y \partial t, \tilde{Z}\right)=-\frac{\partial}{\partial t}\left(a d_{\nabla h(y)(\tilde{l})_{+}}^{*} \tilde{l}, \tilde{Z}\right)+\frac{\partial}{\partial y}\left(a d_{\nabla h^{(t)}(\tilde{l})_{+}} \tilde{l}, \tilde{Z}\right)= \\
& =-\frac{\partial}{\partial t}\left(\tilde{l},\left[\nabla h^{(y)}(\tilde{l})_{+}, \tilde{Z}\right]\right)+\frac{\partial}{\partial y}\left(\tilde{l},\left[\nabla h^{(y)}(\tilde{l})_{+}, \tilde{Z}\right]\right)= \\
& =-\left(\frac{\partial}{\partial t} \tilde{l},\left[\nabla h^{(y)}(\tilde{l})_{+}, \tilde{Z}\right]\right)-\left(\tilde{l},\left[\frac{\partial}{\partial t} \nabla h^{(y)}(\tilde{l})_{+}, \tilde{Z}\right]\right)+ \\
& +\left(\frac{\partial}{\partial y} \tilde{l},\left[\nabla h^{(t)}(\tilde{l})_{+}, \tilde{Z}\right]\right)+\left(\tilde{l},\left[\frac{\partial}{\partial y} \nabla h^{(y)}(\tilde{l})_{+}, \tilde{Z}\right]\right)= \\
& =\left(a d_{\nabla h^{(t)}(\tilde{l})_{+}}^{*} \tilde{l},\left[\nabla h^{(y)}(\tilde{l})_{+}, \tilde{Z}\right]\right)-\left(\tilde{l},\left[\frac{\partial}{\partial t} \nabla h^{(y)}(\tilde{l})_{+}, \tilde{Z}\right]\right)- \\
& -\left(a d_{\nabla h(y)(\tilde{l})_{+}}^{*} \tilde{l},\left[\nabla h^{(t)}(\tilde{l})_{+}, \tilde{Z}\right]\right)+\left(\tilde{l},\left[\frac{\partial}{\partial y} \nabla h^{(y)}(\tilde{l})_{+}, \tilde{Z}\right]\right)= \\
& =\left(\tilde{l},\left[\nabla h^{(t)}(\tilde{l})_{+},\left[\nabla h^{(y)}(\tilde{l})_{+}, \tilde{Z}\right]\right]\right)-\left(\tilde{l},\left[\frac{\partial}{\partial t} \nabla h^{(y)}(\tilde{l})_{+}, \tilde{Z}\right]\right)- \\
& -\left(\tilde{l},\left[\nabla h^{(y)}(\tilde{l})_{+},\left[\nabla h^{(t)}(\tilde{l})_{+}, \tilde{Z}\right]\right]\right)+\left(\tilde{l},\left[\frac{\partial}{\partial y} \nabla h^{(t)}(\tilde{l})_{+}, \tilde{Z}\right]\right)= \\
& =\left(\tilde{l},\left[\nabla h^{(t)}(\tilde{l})_{+},\left[\nabla h^{(y)}(\tilde{l})_{+}, \tilde{Z}\right]\right]-\left[\nabla h^{(y)}(\tilde{l})_{+},\left[\nabla h^{(t)}(\tilde{l})_{+}, \tilde{Z}\right]\right]\right)+ \\
& +\left(\tilde{l},\left[\frac{\partial}{\partial y} \nabla h^{(t)}(\tilde{l})_{+}-\frac{\partial}{\partial t} \nabla h^{(y)}(\tilde{l})_{+}, \tilde{Z}\right]\right)= \\
& =\left(\tilde{l},\left[\left[\nabla h^{(y)}(\tilde{l})_{+}, \nabla h^{(t)}(\tilde{l})_{+}\right]+\frac{\partial}{\partial y} \nabla h^{(t)}(\tilde{l})_{+}-\frac{\partial}{\partial t} \nabla h^{(y)}(\tilde{l})_{+}, \tilde{Z}\right]\right)=\left(a d_{\varphi(\tilde{l})}^{*}, \tilde{l}, \tilde{Z}\right),
\end{aligned}
$$

where we have denoted by

$$
\varphi(\tilde{l}):=\left[\nabla h^{(y)}(\tilde{l})_{+}, \nabla h^{(t)}(\tilde{l})_{+}\right]+\frac{\partial}{\partial y} \nabla h^{(t)}(\tilde{l})_{+}-\frac{\partial}{\partial t} \nabla h^{(y)}(\tilde{l})_{+}
$$

From (75), we obtain that $a d^{*}(\tilde{l})=0$ for all $y, t \in \mathbb{R}, \varphi(\tilde{l}) \in \tilde{\mathcal{G}}$ and arbitrary $\lambda \in \mathbb{C}$. Now, based on the arbitrariness of $\tilde{Z} \in \tilde{\mathcal{G}}$ and analyticity of the $A$-valued vector field expression (76), one easily shows [33] that $\varphi(\tilde{l})=0$, thus finishing the proof.

For the exact representatives of the functions $h^{(y)}, h^{(t)} \in I\left(\tilde{\mathcal{G}}^{*}\right)$, it is necessary to solve the determining equation (66), taking into account that if the chosen element $\tilde{l} \in \tilde{\mathcal{G}}^{*}$ is singular as $|\lambda| \rightarrow \infty$, the related expansion

$$
\nabla h^{(p)}(l) \simeq \lambda^{p} \sum_{j \in \mathbb{Z}_{+}} \nabla h(l)_{j} \lambda^{-j}
$$

where the degree $p \in \mathbb{Z}_{+}$can be taken as arbitrary. Upon substituting (77) into (66), one can find recurrently all the coefficients $\nabla h(l)_{j}, j \in \mathbb{Z}_{+}$, and then construct gradients of the Casimir functions $h^{(y)}, h^{(t)} \in I\left(\tilde{\mathcal{G}}^{*}\right)$ reduced on $\tilde{\mathcal{G}}_{+}$as

$$
\nabla h^{(t)}(l)_{+}=\left(\lambda^{p_{t}} \nabla h(l)\right)_{+}, \quad \nabla h^{(y)}(l)_{+}=\left(\lambda^{p_{y}} \nabla h(l)\right)_{+}
$$

for some positive integers $p_{y}, p_{t} \in \mathbb{Z}_{+}$. 
Remark 14. As mentioned above, the expansion (77) is effective if a chosen seed element $\tilde{l} \in \tilde{\mathcal{G}}^{*}$ is singular as $|\lambda| \rightarrow \infty$. In the case when it is singular as $|\lambda| \rightarrow 0$, the expression (77) should be replaced by the expansion

$$
\nabla h^{(p)}(l) \sim \lambda^{-p} \sum_{j \in \mathbb{Z}_{+}} \nabla h(l)_{j} \lambda^{j}
$$

for an arbitrary $p \in \mathbb{Z}_{+}$, and the reduced Casimir function gradients then are given by the expressions

$$
\nabla h^{(y)}(l)_{-}=\left(\lambda^{-p_{y}-1} \nabla h(l)\right)_{-}, \quad \nabla h^{(t)}(l)_{-}=\left(\lambda^{-p_{t}-1} \nabla h(l)\right)_{-}
$$

for some positive integers $p_{y}, p_{t} \in \mathbb{Z}_{+}$. Then, the corresponding flows are, respectively, written as

$$
\partial \tilde{l} / \partial t=a d_{\nabla h^{(t)}(\tilde{l})_{-}} \tilde{l}, \quad \partial \tilde{l} / \partial y=a d_{\nabla h(y)(\tilde{l})_{-}}^{*} \tilde{l} .
$$

The above results, owing to Lemma 13, can be formulated as the following main proposition.

Proposition 15. Take an A-valued loop vector field $\tilde{l} \in \tilde{\mathcal{G}}^{*}$ and let $h^{(y)}, h^{(t)} \in I\left(\tilde{\mathcal{G}}^{*}\right)$ be Casimir functions subject to the metric $(\cdot, \cdot)$ on the A-valued loop Lie algebra $\tilde{\mathcal{G}}$ and the natural coadjoint action on the A-valued loop co-algebra $\tilde{\mathcal{G}}^{*}$. Then, the following dynamical systems

$$
\partial \tilde{l} / \partial y=-a d_{\nabla h(y)(\tilde{l})_{+}}^{*} \tilde{l}, \quad \partial \tilde{l} / \partial t=-a d_{\nabla h^{(t)}(\tilde{l})_{+}}^{*} \tilde{l}
$$

are commuting to each other Hamiltonian flows for all $y, t \in \mathbb{R}$. Moreover, if $H$ is a faithful representation vector space for the weak Balinsky-Novikov algebra $A$, the compatibility condition of these flows is equivalent to the vector fields representation

$$
\left(\partial / \partial t+\nabla h^{(t)}(\tilde{l})_{+}\right) \psi=0, \quad\left(\partial / \partial y+\nabla h^{(y)}(\tilde{l})_{+}\right) \psi=0,
$$

where $\psi \in C^{2}\left(\mathbb{R}^{2} \times \mathbb{T}^{n} ; H\right)$ and the A-valued loop vector fields $\nabla h^{(t)}(\tilde{l})_{+}, \nabla h^{(y)}(\tilde{l})_{+} \in \tilde{\mathcal{G}}_{+}$, given by the expressions (73) and (78), satisfy the so called Lax-Sato compatible relationship (74) for any $\lambda \in \mathbb{C}$.

\subsection{A Weakly Deformed Balinsky-Novikov Type Symmetry Algebra}

Consider a finite-dimensional noncommutative and non-associative algebra $(A ;+, \cdot)$ over $\mathbb{C}$, endowed additionally with a commutative family of automorphisms $\left\{\Delta_{x}: A \rightarrow A: x \in \mathbb{R}\right\}$, depending smoothly on a real parameter $x$ and satisfying the following weak continuity condition:

$$
\lim _{\varepsilon \rightarrow 0} \Delta_{x+\varepsilon} \Delta_{x}^{-1}=I
$$

for any $x$, which makes it possible to construct a "current algebra"

$$
\mathbb{A}:=\left\{\mathrm{a}(x):=\Delta_{x} a: a \in A, x \in \mathbb{R}\right\}
$$

with the naturally compatible pointwise multiplication:

$$
\mathrm{a}(x) \cdot \mathrm{b}(x):=\Delta_{x} a \cdot \Delta_{x} b=\Delta_{x}(a \cdot b)
$$

for any $\mathrm{a}(x), \mathrm{b}(x) \in \mathbb{A}, x \in \mathbb{R}$. $\mathbb{A}$ can be additionally rigged with the Lie structures:

$$
[\mathrm{a}, \mathrm{b}]_{D}:=(D \mathrm{a}) \cdot \mathrm{b}-(D \mathrm{~b}) \cdot \mathrm{a}
$$

and

$$
[\mathrm{a}, \mathrm{b}]_{D}:=\mathrm{a} \cdot D \mathrm{~b}-\mathrm{b} \cdot D \mathrm{a}
$$


for arbitrary $\mathrm{a}:=\mathrm{a}(x), \mathrm{b}:=\mathrm{b}(x) \in \mathbb{A}$ for any $x \in \mathbb{R}$, where the map

$$
D:=\left(\Delta_{h}-1\right) / h
$$

is an endomorphism $\mathbb{A}$, specified by the parameter $h \in \mathbb{R}$.

Remark 16. It is easy to see that the corresponding operator limit, $\lim _{h \rightarrow 0} D \mathrm{a}(x)=d \mathrm{a}(x) / d x$ for any $\mathrm{a}(x) \in \mathbb{A}$ and $x$.

Now, we pose a problem: what conditions should be imposed on $A$ for $\mathbb{A}$ to become a Lie algebra? For an answer it, is enough to check the Jacobi identity

$$
\left[[\mathrm{a}, \mathrm{b}]_{D}, \mathrm{c}\right]_{D}+\left[[\mathrm{b}, \mathrm{c}]_{D}, \mathrm{a}\right]_{D}+\left[[\mathrm{c}, \mathrm{a}]_{D}, \mathrm{~b}\right]_{D}=0
$$

for any $a, b$ and $c \in \mathbb{A}$.

Observe that mapping (89) satisfies for any $\tilde{a}, \tilde{b} \in \mathbb{A}$ the property

$$
D(\mathbf{a} \cdot \mathbf{b})=D a \cdot b+\mathbf{a}^{(h)} \cdot D \mathbf{b},
$$

where $\mathrm{a}^{(h)}:=\Delta_{h} \mathrm{a} \in \mathbb{A}$. Having defined the usual right $R_{a}$ and left $L_{a}$ shifts on the algebra $A$ as $R_{a} b:=b \cdot a, L_{a} b:=a \cdot b$ for arbitrary $a, b \in A$, respectively, one can easily prove the following result.

Proposition 17. The current algebra $\mathbb{A}_{h}$ is a Lie algebra $\mathcal{L}_{\mathbb{A}_{h}}$ iff the following conditions hold for all $a, b \in A_{h}$ :

$$
\left[L_{a}, L_{b}\right]=L_{\left(\Delta_{h} a\right) \cdot b}-L_{\left(\Delta_{h} b\right) \cdot a}\left[R_{a}, R_{b}\right]=0
$$

for the Lie bracket (87), and

$$
\left[R_{a}, R_{b}\right]=R_{[a, b]}, \quad L_{a} L_{\Delta_{h} b}=L_{b} L_{\Delta_{h} a}
$$

for the Lie bracket (88).

The constraints (92), (93) make it possible to describe $A_{h}$ as a "deformed Balinsky-Novikov type algebra"(dBNA), although it coincides $[17,20]$ as $h \rightarrow 0$ with the classical Balinsky-Novikov algebra $A_{0}$, defined via

$$
\left[L_{a}, L_{b}\right]=L_{[a, b]}, \quad\left[R_{a}, R_{b}\right]=0
$$

and

$$
\left[R_{a}, R_{b}\right]=R_{[a, b]}, \quad\left[L_{a}, L_{b}\right]=0,
$$

respectively, satisfied for any $a, b \in A_{0}$.

As a dBNA $A_{h}$ is assumed to be finite-dimensional, one can naturally determine $[11,13,69]$ the adjoint space $\mathcal{L}_{\mathbb{A}_{h}}^{*}$ to the adjacent current Lie algebra $\mathcal{L}_{\mathbb{A}_{h}}$ as a set of linear continuous functionals $u: \mathcal{L}_{\mathbb{A}_{h}} \rightarrow \mathbb{R}$ on $\mathcal{L}_{\mathbb{A}_{h}}$ via the expression

$$
u(\mathrm{a}):=(u, \mathrm{a})_{s}
$$

for some symmetric bilinear form $(\cdot, \cdot)_{S}$ on $\mathcal{L}_{\mathbb{A}_{h}}$ and to construct on it the canonical Lie-Poisson structure

$$
\{u(\mathrm{a}), u(\mathrm{~b})\}:=u\left([\mathrm{a}, \mathrm{b}]_{D}\right)
$$

for any linear functions $u(\mathrm{a}), u(\mathrm{~b}) \in \mathrm{D}\left(\mathcal{L}_{\mathbb{A}_{h}}^{*}\right), \mathrm{a}, \mathrm{b} \in \mathcal{L}_{\mathbb{A}_{h}}$, with arbitrary $u \in \mathcal{L}_{\mathbb{A}_{h}}^{*}$, satisfying the Jacobi identity owing to (92) and (93). As the expression (96) can be rewritten as

$$
\{u(\mathrm{a}), u(\mathrm{~b})\}:=(\vartheta(u) \mathrm{a})(\mathrm{b})
$$


for any $u \in \mathcal{L}_{\mathbb{A}_{h}}^{*}$ and $\mathrm{a}, \mathrm{b} \in \mathcal{L}_{\mathbb{A}_{h}}$, where the linear map $\vartheta(u): \mathcal{L}_{\mathbb{A}_{h}} \rightarrow \mathcal{L}_{\mathbb{A}_{h}}^{*}$ is called $[11,13,14,17]$ a Hamiltonian operator. This operator makes it possible to construct, for any smooth functional $\gamma \in \mathcal{D}\left(\mathbb{A}_{h}^{*}\right)$, the Hamiltonian system

$$
d u / d t=-\vartheta(u) \operatorname{grad} \gamma(u)
$$

on $\mathcal{L}_{\mathbb{A}_{h}}^{*}$, where $\operatorname{grad} \gamma(u) \in \mathcal{L}_{\mathbb{A}_{h}}$ is the standard gradient of this functional. Moreover, if $\mathcal{L}_{\mathbb{A}_{h}}$ allows the central extension $\hat{\mathcal{L}}_{\mathbb{A}_{h}}:=\left(\mathcal{L}_{\mathbb{A}_{h}} ; \mathbb{C}\right)$ by means of a Maurer-Cartan bilinear form

$$
\omega_{2}(\mathrm{a}, \mathrm{b}):=\left(\mathrm{a}, \alpha\left(\Delta_{h}\right) \mathbf{b}\right)_{s}
$$

where $(\cdot, \cdot)_{s}$ is a symmetric bilinear form on $\mathcal{L}_{\mathbb{A}_{h}}$ and

$$
[(\mathrm{a} ; \alpha),(\mathrm{b} ; \beta)]_{D}:=\left([\mathrm{a}, \mathrm{b}]_{D} ; \omega_{2}(\mathrm{a}, \mathrm{b})\right)
$$

for any $(\mathrm{a} ; \alpha),(\mathrm{b} ; \beta) \in \hat{\mathcal{L}}_{\mathbb{A}_{h}}$ and $\alpha\left(\Delta_{h}\right): \mathbb{A}_{h} \rightarrow \mathbb{A}_{h}$ is some skew-symmetric constant map, then the Hamiltonian operator

$$
\vartheta(u)+\lambda \alpha\left(\Delta_{h}\right),
$$

where $\left(\mathrm{a}, \alpha\left(\Delta_{h}\right) \mathrm{b}\right)_{s}:=\left(\alpha\left(\Delta_{h}\right)^{*} \mathrm{a}, \mathrm{b}\right)_{s}$ for any a, b $\in \mathcal{L}_{\mathbb{A}_{h}}$, is compatible [14] for any $\lambda \in \mathbb{C}$. This makes it possible to generate $[11,13,14]$ an infinite hierarchy of mutually commuting smooth independent functionals $\gamma_{j} \in \mathcal{D}\left(\mathcal{L}_{\mathbb{A}_{h}}^{*}\right), j \in \mathbb{Z}_{+}$, with respect to both $\vartheta(u)$ and $\alpha\left(\Delta_{h}\right)^{*}: \mathcal{L}_{\mathbb{A}_{h}} \rightarrow \mathcal{L}_{\mathbb{A}_{h}}^{*} u \in \mathcal{L}_{\mathbb{A}_{h}}^{*}$ satisfying

$$
\vartheta(u) \nabla \gamma_{j}(u)=\alpha\left(\Delta_{h}\right)^{*} \nabla \gamma_{j+1}(u)
$$

and giving rise to an infinite system of mutually commuting completely integrable Hamiltonian flows

$$
d u / d t_{j}=-\vartheta(u) \operatorname{grad} \gamma_{j}(u)
$$

on $\mathcal{L}_{\mathbb{A}_{h}}^{*}$ with respect to independent evolution parameters $t_{j} \in \mathbb{R}, j \in \mathbb{Z}_{+}$.

\section{The Riemann Type Reduced Pre-Lie Algebra Isomorphism and Related Algebraic Properties}

Observe that $A$ with relationships (50), generated by the two-dimensional toroidal pre-Lie algebra structure (48), is close to the Riemann type pre-Lie algebra structure (47), yet generated by the spatially one-dimensional skew-symmetric structure (46). Moreover, the following result holds.

Theorem 18. The algebra $A$, generated by the relationships (50), is isomorphic to the reduced Riemann type pre-Lie algebra (47).

Let $A=(A,+, \cdot)$ be an algebra,

$$
\begin{gathered}
R(A):=\left\{r_{a} \mid a \in A\right\} \text { and } L(A):=\left\{l_{a} \mid a \in A\right\}, \\
{\left[l_{a}, l_{b}\right]=0=\left[r_{a}, r_{b}\right],} \\
l_{a b}=l_{a} l_{b}
\end{gathered}
$$

and $\left[l_{a}, r_{b}\right]=0$.

Recall that an algebra $A$ is called $a$ Riemann algebra if

$$
(x b) a=(x a) b
$$

and

$$
x(a b)=(a b) x=(b a) x,
$$


and a Balinsky-Novikov algebra if

$$
(a b) c=(a c) b
$$

and

$$
(a b) c-a(b c)=(b a) c-b(a c)
$$

for any $a, b, x \in A$.

In the sequel, we shall denote the center of $A$ by $Z(A)$, the commutator by $[a, b]=a b-b a$ and the commutator subgroup by $[A, A]$.

Lemma 19. If $A$ is an algebra, then the following statements hold:

(i) if A satisfies (104), it is associative,

(ii) if A satisfies (103) and (104), then $[A, A] \cdot A=0$ and $A^{2} \subseteq Z(A)$ (and so $A$ is at most 2-step Lie nilpotent),

(iii) if $A$ satisfies (104) and $A \ni 1$ (respectively $A$ does not contain zero-divisors), then $A$ is commutative,

(iv) if A satisfies (103) and (104), then Equation (50) follows,

(v) if A satisfies (104), then it is a Riemann algebra (and consequently a Novikov-Balinsky algebra).

Proof. Assume that $a, b, x \in A$. For $(i)$, we simply note that

$$
(a b) x=l_{a b}(x)=l_{a} l_{b}(x)=a(b x) .
$$

To verify (ii), we compute that

$$
\begin{aligned}
& a(b x)=l_{a} l_{b}(x)=l_{b} l_{a}(x)=b(a x), \\
& (x b) a=r_{a} r_{b}(x)=r_{b} r_{a}(x)=(x a) b
\end{aligned}
$$

and so

$$
a(b x)=b(a x)=(b a) x=(b x) a,
$$

implying that $A^{2} \subseteq Z(A)$. Moreover, $(a b) x=a(b x)=b(a x)=(b a) x$ in view of (107) and (108). Finally, $[A, A] \subseteq A^{2} \subseteq Z(A)$, so $[[A, A], A]=0$. For (iii), note that, if $b=1$, then (109) implies that $a x=x a$. Then, if $A \neq 0$ and $A$ has no zero-divisors, (ii) implies that $[A, A]=0$. Now, (iv) follows from (103) and (104) and $(v)$ follows from (ii), which completes the proof.

An additive mapping $\delta: A \rightarrow A$ is called a derivation of $A$ if

$$
\delta(a b)=\delta(a) b+a \delta(b)
$$

for all $a, b \in A$. Let $\operatorname{Der} A$ be the set of all derivations of $A$. Moreover,

$$
A^{L}=(A,+,[-,-])
$$

is a Lie algebra, where $[x, y]=x y-y x$ for any $x, y \in A$ (the associated Lie algebra of a Balinsky-Novikov algebra $A$ ). An additive map $\varphi: A \rightarrow A$ is called a Lie derivation of $A$ (or a derivation of $A^{L}$ ) if

$$
\varphi([a, b])=[\varphi(a), b]+[a, \varphi(b)]
$$

for all $a, b \in A$. Letting $\operatorname{Der} A^{L}$, we denote the set of derivations of $A^{L}$. Then, $\operatorname{Der} A \subseteq \operatorname{Der} A^{L}$. A Novikov algebra $A$ is called a derivation algebra if its left multiplications $L_{x}$ or its right multiplication $R_{x}$ are derivations of $A^{L}$ ([28], p. 107). 
Lemma 20. If $A$ satisfies (103) and (104), then: (i) Der $A$ is a left $A$-module; (ii) $L(A), R(A) \subseteq \operatorname{Der} A^{L}$; and (iii) $A$ is a derivation algebra.

Proof. For $(i)$, we note that Lemma $19(i i)$ implies that $[A, A] \cdot \delta(A)=0$ for any $\delta \in \operatorname{Der} A$, so

$$
(a \delta)(x y)=a \delta(x)+a x \delta(y)=a \delta(x)+x a \delta(y)+[a, x] \delta(y)=(a \delta)(x)+x(a \delta)(y)
$$

for any $a, x, y \in A$, so $a \delta \in \operatorname{Der} A$. Hence, (ii) follows as does (iii) in view of [28, Corollary 2.1] and Lemma 19(ii).

\subsection{A General Riemann Type Pre-Lie Algebra Structure}

Let $(A, A)$ be the additive subgroup of $A$ generated by Jordan commutators $(a, b):=a b+b a$, where $a, b \in A$. An associative algebra $D$ is nilpotent if $D^{n}=0$ for some positive integer $n$; the least such $n$ is called the nilpotency index (NI) of $D$.

Lemma 21. If $A$ is a general Riemann type pre-Lie algebra over a field $\mathbb{F}$ satisfying

$$
r_{a} r_{b}=l_{a b}=l_{a} l_{b}
$$

for any $a, b \in A$; then, the following statements hold:

(i) $A$ is associative and $(A,[A, A])=0$,

(ii) $a^{2} \in Z(A)$,

(iii) $(A, A) \subseteq Z(A)$ (in particular, if $A=(A, A)$, then $A$ is commutative),

(iv) if $A$ has unity, then $2[A, A]=0$ (in particular, in $\mathbb{F} \neq 2$, then $A$ is commutative),

(v) if $\mathbb{F} \neq 2$, then $u^{2}=0$ for any $u \in[A, A]$.

Proof. Assume that $a, b, x \in A$; then, (i) follows from

$$
(x a) b=r_{a}\left(r_{b}(x)\right)=l_{a b}(x)=(a b) x
$$

and $(x b) a=r_{a}\left(r_{b}(x)\right)=l_{a}\left(r_{b}(x)\right)=a(b x)$, which imply that $(a b) x=a(b x)$, so $A$ is associative. Moreover,

$$
x(b a)=(x b) a=(a b) x \text { and } x(a b)=(x a) b=(b a) x,
$$

which implies that $(x,[a, b])=0$.

Property (ii) follows from (111) with $b=a$, so $x(a a)=(x a) a=(a a) x$ and therefore $a^{2} \in Z(A)$.

To prove (iii), we use

$$
a^{2}+(a, b)+b^{2}=(a+b)^{2} \in Z(A),
$$

to conclude that $(a, b)$ is central.

Next, $(i v)$ is a consequence of $(i)$ gives that

$$
2 u^{2}=(u, u) \in([A, A],[A, A])=0
$$

for any $u \in[A, A]$ and $(v)$ follows directly from $(i v)$.

Proposition 22. Let $A$ be a non-commutative reduced Riemann type algebra over a field $\mathbb{F}$ satisfying (110) and $\mathbb{F} \neq 2$. Then, the following hold:

(i) if $(A, A)=0$, then $a^{2}=0$ for any $a \in A$ (in particular, if $A$ is finite-dimensional, then $A$ is nilpotent),

(ii) if $(A, A)$ is nonzero proper in $A$, then $A$ is nilpotent with $N I \leq 3$ (and so $A$ is at most 2-step Lie nilpotent).

Proof. If $(A, A)=0$, then $2 a^{2}=(a, a)=0$ for any $a \in A$, which proves $(i)$. 
For $(i i)$, Lemma $21(i)$ implies $([A, A],[A, A])=0$ and so $a b=-b a$ for any $a, b \in[A, A]$. Then,

$$
[[A, A],[A, A]] \ni[a, b]=a b-b a=-2 b a,
$$

which implies

$$
A^{2} \subseteq[[A, A],[A, A]] \subseteq[A, A] \subseteq A^{2}
$$

Consequently, $A^{2}=[A, A]$. In view of Lemma $21(i),\left(A, A^{2}\right)=0$, so

$$
(x y) z=x(y z)=-(y z) x=-y(z x)=(z x) y=z(x y)
$$

for any $x, y, z \in A$. Hence, $A^{2} \subseteq Z(A)$. Since

$$
\left[A, A^{2}\right]=0 \text { and }\left(A, A^{2}\right)=0,
$$

we conclude that $A^{3}=0$. Finally $[[A, A], A] \subseteq A^{3}=0$.

It is evident that a general skew-symmetric integral-differential commutator expression $[a, b]_{D}$ for any $a, b \in \widetilde{A}$ on the $n$-dimensional toroidal algebra $\widetilde{A},(41)$, includes some new BNA-type pre-Lie algebra structures on the basic nonassociative algebra $A$, which can be useful for applications in the multi-dimensional integrability theory started in [32-42] and developed in [43,44]. They are strongly based on differential-algebraic and related analytical techniques and make it possible to construct new algebraic structures on the corresponding nonassociative algebras, within which the corresponding Hamiltonian operators generate integrable multi-component and multidimensional dynamical systems. In what follows, we investigate the underlying algebraic structures of non-associative BNA-type pre-Lie algebras by focusing on the basic Balinsky-Novikov algebra and its fermionic modification.

\section{The Balinsky-Novikov Algebra and Its Fermionic Modification}

Recall that $(N,+, 0)$ is a left-symmetric algebra $(L S A)$, i.e., $(N,+)$ is an $\mathbb{F}$-linear space with a bilinear product $(x, y) \mapsto x y:=x \circ y$ satisfying (38) for all $a, b, c \in N$. Every BNA is an LSA. Moreover,

$$
N^{L}=(N,+,[-,-])
$$

is a Lie algebra, where $[x, y]=x y-y x$ for any $x, y \in N$ ( the associated Lie algebra of an LSA N). LSAs play a fundamental role in theory of affine manifolds (cf. [70]). Obviously, $N^{L}$ is abelian if and only if $N$ is abelian. An algebra $N$ satisfying (38) and

$$
(a b) c=-(a c) b
$$

modifying (37) for all $a, b, c \in N$, is called a fermionic BNA. A (nonassociative or associative) algebra $A$ is called: semiprime if, for any ideal $T$ of $A$, the condition $T^{2}=0$ implies that $T=0$; prime if, for any ideals $T, Q$ of $A$, the condition $T Q=0$ implies that $T=0$ or $Q=0$; and simple if $A^{2} \neq 0$ and its only ideals are 0 and $A$.

It is easy to see that every simple BNA is prime and every prime BNA is semiprime. The theory of BNA's was started by Zelmanov [59]. He proved that a finite-dimensional simple BNA over an algebraically closed field of characteristic 0 is one-dimensional. Osborn [71] proved that, for any finite-dimensional simple BNA $N$ over a perfect field of characteristic $p>2$, the associated Lie algebra $N^{L}$ is isomorphic to the rank-one Witt algebra. Simple BNA's have also been investigated by Zelmanov, Osborn and $\mathrm{Xu}$ [71-73]. Many authors have investigated the Lie structure of BNA's. BNA's $N$ with abelian (respectively nilpotent, solvable) associated Lie algebras $N^{L}$ have been studied by Burde and de Graaf, Burde, Dekimpe and Vercammen, Burde and Dekimpe [74-76]. The class of commutative associative algebras (CAA) equals the class of BNA's with abelian associated Lie algebras. CAA's (real and complex) of dimension 3 were characterized in [76] (see, e.g., Baehr, Dimakis and 
Müller-Hoisson [77]) and Balinsky-Novikov $\mathbb{C}$-algebras $N$ of dimension 4 with the nilpotent associated Lie algebras $N^{L}$. In [70], it is proved that a complete LSA is always solvable. Recall that a BNA $N$ is complete (or transitive) if the right multiplication operator $r_{a}$ is a nilpotent linear map for any $a \in N$. Burde [78] investigated a Lie algebra $N^{L}$ of a simple LSA $N$. We investigate properties of semiprime BNA's (see Proposition 36) leading to a proof of the following result.

Theorem 23. Let $N$ be a BNA. Then, the following statements hold:

(1) Suppose that $N$ is non-commutative and char $\mathbb{F} \neq 2$. If $N$ is simple (respectively prime), then it contains a commutative Lie ideal A that contains every commutative Lie ideal of $N$ and $N^{L} / A$ is a simple (respectively prime) Lie algebra.

(2) If $N^{L}$ is a simple (respectively prime or semiprime) Lie algebra, then $N$ is a simple (respectively prime or semiprime) BNA.

Let

$$
R(N):=\left\{r_{a} \mid a \in N\right\} \text { and } L(N):=\left\{l_{a} \mid a \in N\right\}
$$

and $\mathcal{R}(N)$ be the Lie algebra generated by $R(N)$. If $N$ is a fermionic BNA and $R_{1}:=R(N), R_{i+1}:=$ $\left[R_{1}, R_{i}\right]$, then

$$
\mathcal{R}(N)=R_{1}+\cdots+R_{i}+\cdots=R_{1}+R_{2}=R(N)+R(N) R(N)
$$

(see [28], Claim 1). Let $\mathcal{L}(N)$ be the Lie algebra generated by all $r_{x}$ and $l_{y}$, where $x, y \in N$. By [28] (Claim 2):

$$
\mathcal{L}(N)=L(N)+R(N)+R(N) R(N) .
$$

Let $A R(N)$ denote an associative algebra generated by $R(N)$ (with respect to two operations: addition and composition of operators). Moreover, left multiplication operators of a BNA $N$ forms a Lie algebra $L(N)$ with respect to the pointwise addition "+ "and the pointwise Lie multiplication " $[-,-]$ "given by the rules

$$
\left(l_{a}+l_{b}\right)(x)=l_{a}(x)+l_{b}(x) \text { and }\left[l_{a}, l_{b}\right](x)=l_{a}\left(l_{b}(x)\right)-l_{b}\left(l_{a}(x)\right)
$$

for all $a, b, x \in N$. A map $\delta: N^{L} \rightarrow N^{L}$ is called a derivation of $N^{L}$ if

$$
\delta(a+b)=\delta(a)+\delta(b) \text { and } \delta([a, b])=[\delta(a), b]+[a, \delta(b)]
$$

for all $a, b \in N^{L}$. The set $\operatorname{Der}\left(N^{L}\right)$ of all derivations of $N^{L}$ is a Lie algebra over $\mathbb{F}$. An LSA $N$ is $a$ derivation algebra if its left multiplications $l_{x}$ or its right multiplications $r_{x}$ are derivations [27] of the associated Lie algebra $N^{L}$. By [27] (Corollary 2.1), a BNA $N$ is a derivation algebra if and only if $[N, N] \subseteq$ lann $N$ (and so $N$ is at most 2-step nilpotent). We have the following result.

Proposition 24. Let $N$ be a fermionic BNA. Then, the following statements hold:

(i) the Lie algebra $\mathcal{L}(N)=L(N)+X_{N}$ is a sum of a subalgebra $L(N)$ and an ideal $X_{N}:=R(N)+$ $R(N) R(N)$, where $L(N)$ and $N^{L} /$ lann $N$ are isomorphic, where lann $N:=\{x \in N \mid x N=0\}$ and $X_{N}$ is at most 2-step Lie nilpotent,

(ii) if $L(N) \subseteq \operatorname{Der}\left(N^{L}\right)$, then $[N, N] \subseteq \operatorname{lann} N$ and $\mathcal{L}(N)=L(N)+R(N) \subseteq \operatorname{Der}\left(N^{L}\right)$.

Jacobson [79] initiated an investigation of (associative) multiplicative algebras of nonassociative finite-dimensional algebras $A$ (see e.g., [80,81] and others). Bai and Meng [25] classified complete Balinsky-Novikov $\mathbb{C}$-algebras with nilpotent associated algebras. Recall that an LSA $A$ is right-nilpotent of length $\leq n$, where $n \geq 1$ is a fixed integer, if $r_{a_{1}} r_{a_{2}} \ldots r_{a_{n-1}}\left(a_{n}\right)=0$ for all $a_{1}, a_{2}, \ldots, a_{n-1}, a_{n} \in A$. 
By [82] (Theorem 2), a BNA $N$ of bounded index over a field of characteristic 0 is nilpotent. It is also known [28] (Corollary 1) that every fermionic BNA $N$ is right-nilpotent because

$$
r_{x}^{2}=0 \text { and } r_{x} r_{y}=-r_{y} r_{x}
$$

for any $x, y \in N$. We shall prove that, in any finite-dimensional fermionic BNA $N$ over $\mathbb{F}$ of characteristic $\neq 2, A R(N)$ is a finite-dimensional nilpotent associative algebra of $N I \leq 1+\operatorname{dim}_{\mathbb{F}} N$ (see Proposition 31).

All other definitions and facts are can be found in [83-85].

\section{Elementary Properties of Fermionic BNA's}

Here, $(a, b):=a b+b a$ for any $a, b \in N$ and $(V, V)$ is an additive subgroup of $N$ generated by the set $\{(u, v) \mid u, v \in V\}$, where $V \subseteq N$. As usual, an additive subgroup $A$ of an algebra $N$ is called $a n$ ideal if $A N, N A \subseteq N$. An additive subgroup $U$ of a BNA $N$ is a Lie ideal of $N$ if $[U, N] \subseteq U$. Clearly, $U$ is a Lie ideal of $N$ if and only if $U$ is an ideal of the associated Lie algebra $N^{L}$. We shall need the following analogs of [75] (Lemmas 2.1,2.2,2.7).

Lemma 25. Let $N$ be a fermionic BNA, with ideals I and J. Then: (i) $Z(N)$ is an ideal of $N$; (ii) if $U$ is a Lie ideal of $N$, then $Z(U)$ is the units; (iii) IJ is an ideal of $N$; and (iv) if I is commutative, then $I^{2} \subseteq Z(N)$.

Proof. Assume that $x, y \in N, z \in Z(N), i, t \in I$ and $j \in J$.

Property $(i)$ follows from

$$
\begin{gathered}
x(z y)=z(y x)+(y z) x-(z y) x=z(x y)= \\
\quad=(x y) z=-(x z) y=-(z x) y=(z y) x
\end{gathered}
$$

and $x(y z)=x(z y)=(z y) x=(y z) x$, which implies that $y z, z y \in Z(N)$.

In fact, if $u \in U$, then $[[z, x], u]=-[[x, u], z]-[[u, z], x]=0$ and so $[z, x] \in Z(U)$, which proves (ii).

The proof of (iii) follows from $x(i j)=(x i) j+i(x j)-(i x) j \in I J$ and $(i j) x=-(i x) j \in I J$, and $(i v)$ is a direct consequence of

$$
\begin{gathered}
0=[i, x t]=i(x t)-(x t) i=x(i t)+(i x) t-(x i) t-(x t) i= \\
=x(i t)+(i x) t=x(i t)-(i t) x=[x, i t] .
\end{gathered}
$$

Every ideal of a BNA $N$ is a Lie ideal of $N$. An ideal $B$ of $N$ is called non-central if $B \nsubseteq Z(N)$.

Lemma 26. If $N$ is a fermionic $B N A$, with ideal $A$ and Lie ideal $U$, then: (i) the left annihilator lann $A:=$ $\{n \in N \mid n A=0\}$ of $A$ in $N$ is an ideal of $N$; (ii) if $\operatorname{char} \mathbb{F} \neq 2$, then $I_{N}(U):=\{u \in U \mid u N+N u \subseteq U\}$ is an ideal of $N$ and $I_{N}(U) \subseteq U$; and (iii) the centralizer $C_{N}(U):=\{z \in N \mid z u=u z$ for any $u \in U\}$ of $U$ in $N$ is a Lie ideal of $N$.

Proof. Assume that $n, t, x \in N$. If $a \in A$ and $b \in \operatorname{lann} A$, then $(b n) a=-(b a) n=0$ and

$$
(n b) a=n(b a)-b(n a)+(b n) a=(b n) a=-(b a) n=0,
$$

which proves $(i)$.

To prove (ii), we note that if $b \in I_{N}(U)$ and $N$ is a fermionic BNA, then $b n, n b \in U$ and $(n b) t=$ $-(n t) b,[t, n b] \in U$ and so $t(n b) \in U$. Hence, $n b \in I_{N}(U)$. Since $x(b n)-(b n) x=[x, b n] \in U$ and $x(b n)+(b n) x=x(b n)+(b x) n=b(x n)+(x b) n-(b x) n+(b x) n=b(x n)-(x n) b \in U$, we conclude that $x(b n),(b n) x \in U$, so $b n \in I_{N}(U)$.

Finally, inasmuch as $[[c, n], u]=-[[n, u], c]-[[u, c], n]=0$ for any $c \in C_{N}(U), u \in U$, we deduce that $[c, n] \in C_{N}(U)$, which proves (iii). 
Lemma 27. Let $N$ be a fermionic $B N A, U$ its Lie ideal and $I, J$ its subsets. Then: (i) if $[I, J]=0$, then $(I, I) J=0$ (and consequently $(N, N) Z(N)=0$ ); (ii) if $[I, I]=0$ and char $\mathbb{F} \neq 2$, then $\left(I^{2}\right) I=0$; (iii) if $[I, I]=0$ and char $\mathbb{F} \neq 2$, then $\left(I^{2}\right) Z(N)=0$; and (iv) $\left(C_{N}(U), C_{N}(U)\right) U=0$ and $(U, U) C_{N}(U)=0$.

Proof. Let $x, y \in N, a, b \in I$ and $c \in J$. We have $(a b) c=-(a c) b=-(c a) b=(c b) a=(b c) a=-(b a) c$ and so $(a b+b a) c=0$, which proves $(i)$

Next, $(i)$ implies $2 a b=[a, b]+(a, b) \in$ lann $I$ for any $a, b \in I$, which verifies (ii).

For (iii), from $(a b+b a) Z(N)=0$ and $(a b-b a) Z(N)=0$, we obtain $(2 a b) Z(N)=0$ and the result follows.

As $\left[C_{N}(U), U\right]=0,(i v)$ follows in view of $(i)$.

Corollary 28. Let $N$ be a fermionic BNA and $a \in N$. Then: (i) every commutative subalgebra I of $N$ is nilpotent and $I^{3}=0$ (and so $\mathrm{Z}(\mathrm{N})^{3}=0$ ); and (ii) $\mathrm{Na}$ is a commutative right ideal of $\mathrm{N}$ and $(\mathrm{Na})^{3}=0$.

Proof. Property (i) follows from Lemma 27(ii), and (ii) is a direct consequence of $(x a)(y a)=$ $y((x a) a)+((x a) y) a-(y(x a)) a=(y a)(x a)$ and $(x a) y=-(x y) a \in N a$ for any $x, y \in N$.

Lemma 29. Let $N$ be $a$ LSA and $a, b \in N$. Then: $(i)\left[l_{a}, l_{b}\right]=l_{[a, b]}$; (ii) if $B$ is a Lie ideal of $N$, then $L_{B}(N):=\left\{l_{b} \mid b \in B\right\}$ is an ideal of $L(N)$ (in particular, $L(N)=L_{N}(N)$ ); (iii) $l_{a}=0$ iff $a \in$ lann $N$; (iv) if $\Phi$ is an ideal of the Lie ring $L(N)$, then $\Delta_{\Phi}=\left\{a \in N \mid l_{a} \in \Phi\right\}$ is a Lie ideal of $N$; (v) if $\Phi$ is an ideal of $L(N), \Phi=L_{\Delta_{\Phi}}(N)$; and (vi) there is a Lie algebra isomorphism

$$
L(N) \ni l_{x} \mapsto x+\operatorname{lann} N \in N^{L} / \text { lann } N .
$$

Proof. Verification of $(i),(i i i),(v)$ and $(v i)$ is straightforward.

If $a, b \in B$ and $r \in N$, then $a-b,[a, r] \in B$ and so $l_{a}-l_{b}=l_{a-b},\left[l_{a}, l_{r}\right]=l_{[a, r]} \in L_{B}(N)$, which proves (ii)

Property (iv) follows from the fact that $l_{a}, l_{b} \in \Phi$ and $l_{r} \in L(N)$, then $l_{a-b}=l_{a}-l_{b}, l_{[a, r]}=$ $\left[l_{a}, l_{r}\right] \in \Phi$ and therefore $a-b,[a, r] \in \Delta_{\Phi}$.

Lemma 30. If $B$ is a left ideal of a fermionic $B N A N$, then

$$
R_{B}(N)+R_{B}(N) R(N):=\left\{r_{b}+\sum_{x, t} r_{t} r_{x} \text { is a finite sum } \mid b, t \in B, x \in N\right\}
$$

is an ideal of the Lie algebra $\mathcal{L}(N)$ at most 2-step Lie nilpotent.

Proof. Let $N$ be a fermionic BNA and $a, x, y \in N$. Then, (see [28]),

$$
\begin{gathered}
{\left[r_{a}, r_{y}\right]=2 r_{a} r_{y},} \\
{\left[r_{a}, r_{x} r_{y}\right]=0,} \\
{\left[l_{x}, r_{a}\right]=r_{x a}-r_{a} r_{x},} \\
{\left[l_{x}, r_{a} r_{y}\right]=r_{a} r_{x y}+r_{x a} r_{y} .}
\end{gathered}
$$

If $a \in B$, then $x a \in B$ and the assertion holds.

Proposition 31. If $N$ is a finite-dimensional fermionic $B N A$ over $\mathbb{F}, A R(N)$ is a finite-dimensional nilpotent algebra of $N I \leq 1+\operatorname{dim}_{\mathbb{F}} N$. 
Proof. Let $\left(e_{1}, \ldots, e_{m}\right)$ be a basis of the $\mathbb{F}$-linear space $N$ and $r_{i}:=r_{e_{i}}(i=1, \ldots, m)$. Every $b=$ $b_{1}+\cdots+b_{q} \in A R(N)$ is a finite sum of summands of the form

$$
b_{l}=\alpha_{l} r_{a_{1}} \cdots r_{a_{t_{l}}}=\prod_{j=1}^{t_{l}} \sum_{i=1}^{m} \beta_{i j} r_{i}(1 \leq l \leq q)
$$

for some $\beta_{i j} \in \mathbb{F}$, where $\alpha_{l} \in \mathbb{F}$ and $a_{j} \in N(j=1, \ldots, t)$. If $s$ is a positive integer,

$$
b^{s}=\sum_{\substack{s_{1}+\cdots+s_{q}=s \\ s_{1} \geq 0, \ldots, s_{q} \geq 0}} \pm \frac{s !}{s_{1} ! \cdots s_{q} !} b_{1}^{s_{1}} \cdots b_{q}^{s_{q}} .
$$

Moreover,

$$
\begin{gathered}
b_{l}^{s_{l}}= \pm \prod_{j=1}^{t_{l}}\left(\sum_{i=1}^{m} \beta_{i j} r_{i}\right)^{s_{l}}= \\
= \pm \prod_{j=1}^{t_{l}} \sum \begin{array}{c}
k_{1}+\cdots+k_{m}=s_{l} \quad \frac{s_{l} !}{k_{1} ! \cdots k_{m} !}\left(\beta_{1 j} r_{1}\right)^{k_{1}} \cdots\left(\beta_{m j} r_{m}\right)^{k_{m}} . \\
k_{1} \geq 0, \ldots, k_{m} \geq 0
\end{array}
\end{gathered}
$$

If $s>m$, there exists an integer $p(1 \leq p \leq q)$ such that $s_{p}>1$, so there is an integer $h(1 \leq h \leq m)$ such that

$$
k_{h} \geq 2 \text {. }
$$

Thus, $b^{s}=0$. By [86, Theorem], $A R(N)$ is a nilpotent algebra. Obviously, $A R(N)$ is finite-dimensional.

Proof of Proposition 24. Let $a, x, y, z, t \in N$.

To prove (i), observing from (115)-(118), it follows that

$$
\left[r_{a} r_{x}, r_{y} r_{z}\right](t)=r_{a} r_{x} r_{y} r_{z}(t)-r_{y} r_{z} r_{a} r_{x}(t)=0
$$

and so

$$
\left[X_{N}, X_{N}\right]=[R(N), R(N)]+[R(N) R(N), R(N) R(N)] \subseteq R(N) R(N),
$$

implying $\left[\left[X_{N}, X_{N}\right], X_{N}\right]=0$. The rest holds in view of (113) and Lemmas 29 and 30.

For (ii), we see that if $l_{a} \in \operatorname{Der}\left(N^{L}\right)$, then

$$
\begin{gathered}
(a x) y-y(a x)+x(a y)-(a y) x=\left[l_{a}(x), y\right]+\left[x, l_{a}(y)\right]=l_{a}([x, y])= \\
=a(x y-y x)=a(x y)-a(y x)= \\
=x(a y)+(a x) y-(x a) y-y(a x)-(a y) x+(y a) x
\end{gathered}
$$

and we have $(y a) x=(x a) y$. Hence, $l_{y a}=r_{y} r_{a}$ and, owing to [28] (Claim 2) $\mathcal{L}(N)=L(N)+R(N)$. Since $R(N) \subseteq \operatorname{Der}\left(N^{L}\right)$, the assertion follows.

\section{Lie Structure of Semiprime BNA's}

Lemma 32. If $N$ is a $B N A$ and $\operatorname{char} \mathbb{F} \neq 2$, then $Z(N),[N, N]$ are ideals of $N$ and $[N, N] Z(N)=0$.

Proof. For a proof, see [75] (Lemmas 2.3, 2.6, 2.7).

An additive subgroup $U$ of a BNA $N$ is a Lie ideal of $N$ if $[U, N] \subseteq U$. Clearly, $U$ is a Lie ideal of $N$ iff $U$ is an ideal of $N^{L}$. Every ideal of $N$ is a Lie ideal of $N$. An ideal $B$ of $N$ is called non-central if $B \nsubseteq Z(N)$.

Lemma 33. Let $N$ be a BNA, $A$ its ideal and $U$ its Lie ideal. Then:

(i) the left annihilator lann $A:=\{n \in N \mid n A=0\}$ of $A$ in $N$ is an ideal of $N$,

(ii) if $\operatorname{char} \mathbb{F} \neq 2$, then $I_{N}(U):=\{u \in U \mid u N+N u \subseteq U\}$ is an ideal of $N$ and $I_{N}(U) \subseteq U$, 
(iii) if char $\mathbb{F} \neq 2$, then $[U, U]=0$ or $U$ contains a non-central ideal of $N$,

(iv) if $z \in Z(N)$, then $z N:=\{z n \mid n \in N\}$ is an ideal of $N$,

(v) $T(U):=\{x \in N \mid[x, N] \subseteq U\}$ is a Lie ideal of $N$ and $U \subseteq T(U)$,

(vi) $Z(U)$ is a Lie ideal of $N$,

(vii) $Z(A)$ is an ideal of $N$,

(viii) the centralizer $C_{N}(A):=\{z \in N \mid z a=a z$ for any $a \in A\}$ of $A$ is an ideal of $N$,

(ix) $C_{N}(U)$ is a Lie ideal of $N$,

$(x)$ if $N$ is prime, then $Z(N)=0$ or it is an associative and commutative domain.

Proof. Assume that $n, x, y \in N, u, v \in U, a \in A$. Let $b \in$ lann $A$. Then, (i) follows from ( $b n) a=$ $(b a) n=0$ and

$$
(n b) a=n(b a)-b(n a)+(b n) a=(b n) a=(b a) n=0 .
$$

Since $b n, n b \in U$ for any $b \in I_{N}(U)$ and $(n b) x=(n x) b,[x, n b] \in U$, we conclude that $x(n b) \in U$ what means that $n b \in I_{N}(U)$. Moreover,

$$
x(b n)+(b n) x=x(b n)+(b x) n=b(x n)+(x b) n=b(x n)+x(n b) \in U
$$

and $x(b n)=(b n) x=[x, b n] \in U$ and so $2 x(b n) \in U$. Thus, $x(b n) \in U$ and $(b n) x \in U$ by (119). Hence $b n \in I_{N}(U)$, which proves $(i i)$.

To prove (iii), assume that $[u, v] \neq 0$ for some $u, v \in U$. Then,

$$
\begin{gathered}
{[u, v x]=u(v x)-(v x) u=} \\
=v(u x)+(u v) x-(v u) x-(v x) u= \\
=[u, v] x+v(u x)-(v u) x-(u x) v+(u x) v= \\
=[u, v] x+[v, u x]+[u, v] x,
\end{gathered}
$$

which implies that $[u, v] x \in U$. Inasmuch as $[u, v] x-x[u, v]=[[u, v], x] \in U$, it follows that $x[u, v] \in U$. This yields $0 \neq[u, v] \in I_{N}(U)$.

Property (iv) follows from the fact, $z n \in Z(N)$ for any $z \in Z(N)$ by Lemma 32, so $x(z n)=$ $(z n) x=(n z) x=(n x) z=z(n x) \in z N$.

If $t \in T(U)$, then $[t, x] \in U \subseteq T(U)$, which proves $(v)$.

To verify $(v i)$, simply observe that $[[z, n], a]=-[[n, a], z]-[[a, z], n]=0$ for any $z \in Z(A)$ implies that $[z, n] \in Z(A)$.

For (vii), we see that

$$
(z n) a=(z a) n=(a z) n=(a n) z=z(a n)=a(z n)+[z, a] n=a(z n)
$$

for any $z \in Z(A)$, so $z n \in Z(A)$. Then, $n z=[n, z]+z n \in Z(A)$ owing to (vi).

Property (viii) follows from the fact that, if $c \in C_{N}(A)$, then

$$
(c x) a=(c a) x=(a c) x=(a x) c=c(a x)=a(c x)+[c, a] x=a(c x)
$$

and

$$
\begin{gathered}
(x c) a=(x a) c=c(x a)=x(c a)+[c, x] a= \\
=x(a c)+[c, x] a=a(x c)+[x, a] c+[c, x] a=a(x c) .
\end{gathered}
$$

Then, we have that $[[c, x], u]=-[[x, u], c]-[[u, c], x]=0$ for any $c \in C_{N}(U)$, which proves $(i x)$.

The proof of $(x)$ follows since Lemma 32 implies $[N, N] Z(N)=0$. Consequently, if $Z(N) \neq 0$, $[N, N]=0$ and $(x y) n=(y x) n=(y n) x=x(y n)$. Hence, $N$ is associative. 
In [26] (p. 10056), lann $N$ is called the kernel ideal of an LSA $N$.

Lemma 34. Let $N$ be a BNA, I, J its nonzero ideals such that $[I, J]=0$ and char $\mathbb{F} \neq 2$. Then: (i) $([N, N] J) I=0$; and (ii) if, moreover, $N$ is prime, then it is commutative.

Proof. Assume that $a, b \in N, i \in I$ and $j \in J$. Then,

$$
\begin{aligned}
& (b i)(a j)+([a, b] j) i=(b(a j)) i+([a, b] j) i=(a(b j)) i=(a i)(b j)= \\
& =(b j)(a i)=(b(a i)) j=(a(b i)) j+([b, a] i) j=(a j)(b i)+([b, a] i) j
\end{aligned}
$$

implies that $2([a, b] j) i=0$ and therefore $([N, N] J) I=0$. If $N$ is prime, then $[N, N] J=0$, so $[N, N]=0$.

Lemma 35. Let $A, B$ be ideals of a semiprime $B N A N$. Then: (i) if $A B=0, B A=0$; (ii) lann $A \subseteq \operatorname{rann} A$, where $\operatorname{rann} N:=\{x \in N \mid N x=0\}$; (iii) $A \cap \operatorname{lann} A=0$; and (iv) $\operatorname{rann} N=0=\operatorname{lann} N$.

Proof. If $A B=0,(B A)^{2}=(B A)(B A) \subseteq A B=0$ and, by the semiprimeness of $N, B A=0$ and this proves $(i)$.

Property (ii) follows directly since lann $A$ is an ideal of $N$ by Lemma 26(ii) and $(A \cdot(\operatorname{lann} A))^{2}=$ 0 , so $A \cdot(\operatorname{lann} A)=0$.

Properties (iii) and (vi) are self-evident.

Proposition 36. Let $N$ be a semiprime BNA, I its ideal, $A$ its Lie ideal. Then: (i) if $[I, I] \neq 0$, then $[I, I] \cap Z(N)=0$; (ii) if $[I, I]=0$, then $I \subseteq Z(N)$; (iii) if $[A, A] \subseteq Z(N)$, then $A$ is commutative; (iv) $Z(N / Z(N))=0 ;(v) N / Z(N)$ is a semiprime $B N A$; and (vi) $Z(I) \subseteq Z(N)$.

Proof. Property (i) is obvious in view of Lemma 32. For (ii), assume that $a, b \in N$ and $i, j \in I$. By Lemma 34, $([N, N] I) I=0$. This yields $([N, N] I)^{2}=0$ and therefore $[N, N] I=0$. Consequently, $[I, N]^{2}=0$ and, by the semiprimeness, $[I, N]=0$.

Assume that $a, b \in A, m, n \in N$ and $z \in[A, A]$. Then, $z^{2}=0$ by Lemma 32 and $z N$ is an ideal of $N$ by Lemma 33(iv). Since $z n \in Z(N)$ by Lemma 32 and

$$
\begin{gathered}
(z n)(z m)=z((z n) m)+((z n) z) m-(z(z n)) m= \\
=z((z n) m)=((z n) m) z=((z n) z) m=\left(z^{2} n\right) m=0,
\end{gathered}
$$

we deduce that $(z N)^{2}=0$. This yields that $z \in$ ann $N=0$. Consequently, $[A, A]=0$, thus proving (iii). If $Z_{2}$ is an inverse image of $Z(N / Z(N))$ in $N,\left[N, Z_{2}\right] \subseteq Z(N)$. Then, $Z_{2}$ is an ideal of $N$ and $\left[N, Z_{2}\right]^{2}=0$ by Lemma 32. Hence, $Z_{2}=Z(N)$, which proves $(i v)$.

For $(v)$, we note that, if $\bar{A}$ is an ideal of $N / Z(N)$ such that $\bar{A}^{2}=\overline{0}$ and $A$ is its inverse image in $N$, then $A^{2} \subseteq Z(N)$. Hence, $[A, A] \subseteq Z(N)$ and so $A$ is central by (ii) and (iii).

Finally, (vi) follows from (ii) and (iii).

Proof of Theorem 23. To prove (1), we first note that the hypotheses imply that $[N, N]$ is a nonzero ideal of $N$.

(a) Assume that $N$ is a simple BNA and $U$ is a nonzero proper Lie ideal of $N$. Then, $Z(N)=0$ by Lemma 32 and $N=[N, N]$. By Lemma 26(v), $[U, U]=0$ in view of Lemma 33(iii). Let $C:=C_{N}(U)$. Then, $C$ is a proper Lie ideal of $N$.

$\left(a_{1}\right)$ If $[C, C]=N$, then $C=N$ and so $U$ is central, a contradiction. Hence, $C$ is commutative.

$\left(a_{2}\right)$ Assume that $V$ is a commutative Lie ideal of $N$. If $V \cap U=0$, then $V \subseteq C$. Assume that $V \cap U$ is nonzero and $C_{1}:=C_{N}(V \cap U)$. As in $\left.a_{1}\right), C_{1} \neq N$ and $C_{1}$ is commutative by Lemma 33(iii). Since $U \subseteq C \subseteq C_{1}$, we deduce that $C=C_{1}$. Then, $V \subseteq C$ and consequently $C$ contains all commutative Lie ideals of $N$. Hence, $N^{L} / C$ is a simple Lie algebra. 
(b) Let $N$ be a prime BNA and $A, B$ be nonzero Lie ideals of $N$ such that $[A, B]=0$. If $[A, A] \neq 0$ and $[B, B] \neq 0$, then, by Lemma 33(iii), there exist non-central ideals $A_{0}$ and $B_{0}$ such that $A_{0} \subseteq A$ and $B_{0} \subseteq B$ and $\left[A_{0}, B_{0}\right]=0$, contradicting Lemma 34(i). Therefore, we assume that $A$ is commutative. Let $C:=C_{N}(A)$. If $T(C)=N$, then $[N, N] \subseteq C$, which leads to a contradiction in view of Lemma 34 . Hence, $T(C)$ is proper in $N$. If $[T(C), T(C)] \neq 0, T(C)$ contains a non-central ideal $I_{0}$ of $N$ by Lemma 34(iii) and so $A \subseteq C_{N}\left(\left[I_{0}, N\right]\right)$. Since $C_{N}\left(\left[I_{0}, N\right]\right)$ is an ideal of $N$ by Lemmas 32 and 33(viii), we obtain a contradiction in view of Lemma 34. Thus, $T(C)$ is commutative and $C=T(C)$.

Let $K$ an arbitrary Lie ideal of $N$ such that $[K, K]=0$. If $K \cap A=0$, then $K \subseteq C$. Assume that $K \cap A$ is nonzero and $C_{1}:=C_{N}(K \cap A)$. As above, $C_{1}=T\left(C_{1}\right)$ is commutative and therefore $C_{1}=C$. Hence, $N^{L} / C$ is a prime Lie algebra.

We prove (2) as follows: (a) Assume that $N^{L}$ is a simple Lie algebra. Then, (2) follows because every ideal of $N$ is its Lie ideal.

(b) Let $N^{L}$ be a prime Lie algebra and $A, B$ be ideals of $N$ such that $A B=0$. Since $[A, B] \subseteq A \cap B$ and $[A \cap B, A \cap B] \subseteq A B,[A, B]=0, A=0$ or $B=0$.

(c) If $A$ is a nonzero ideal of $N$ and $N^{L}$ is semiprime, then $A^{2} \neq 0$ because $[A, A] \subseteq A^{2}$. Thus, the proof is complete.

\section{Conclusions}

We proved that an algorithm based on Lie-Poisson structure analysis on the adjoint space to toroidal Lie algebras allows for constructing new algebraic structures within which the corresponding Hamiltonian operators exist and generate integrable multi-component and multidimensional dynamical systems. We also showed that the well-known Balinsky-Novikov algebraic structure, obtained as a condition for a matrix differential expression to be Hamiltonian, arises in our approach as a derivation on the Lie algebra, naturally associated with a differential loop algebra. Using the theory of nonassociative and associative left-symmetric algebras, we described algebraic properties of new Balinsky-Novikov type algebras, including their fermionic version and important related multiplicative and Lie structures.

Author Contributions: The research was a joint venture of four coauthors within which the project administartion, review and editing as well as main conceptualization and methodology were suggested and done by D.B., formal differential-algebraic analysis, analytical performance, as well as original draft peraparation, were completed by A.A.B. and A.K.P., the conceptual extension, references data and algebraic structures analysis, as well as checking the main inferences, were performed mainly by O.D.A.

Funding: This research received no external funding.

Acknowledgments: The authors cordially thank Maciej Błaszak, Jan Cieślinski, Antoni Sym and Anatolij Samoilenko for their cooperation and useful discussions during the International Conference in Functional Analysis dedicated to the 125th anniversary of Stefan Banach held on 18-23 September 2017 in Lviv, Ukraine. A.P. thanks also the Department of Mathematical Sciences of the New Jersey Institute of Technology, (Newark, NJ, USA) for the invitation to visit the New Jersey Institute of Technology, during the summer of 2017, where an essential part of this paper was formulated. A.P. thanks the Department of Physics, Mathematics and Computer Science of the Cracov University of Technology for a local research grant F-2/370/2018/DS.

Conflicts of Interest: The authors declare no conflict of interest.

\section{References}

1. Chapoton, F.; Livernet, M. Pre-Lie algebras and the rooted trees operad. Int. Math. Res. Not. 2001, $395-408$. [CrossRef]

2. Szczesny, M. Pre-Lie algebras and indicence categories of colored rooted trees. arXiv 2010, arXiv:1007.4784v1.

3. Vinberg, E.B. The theory of homogeneous convex cones. Transl. Moscow Math. Soc. 1963, 12, 340403.

4. Matsushima, Y. Affine structures on complex manifolds. Osaka J. Math. 1968, 5, 215-222.

5. Gerstenhaber, M. The cohomology structure of an associative ring. Ann. Math. 1963, 78, 267-288. [CrossRef]

6. Kreimer, D. On the Hopf algebra structure of perturbative quantum field theory. Adv. Theor. Math. Phys. 1998, 2, 303-334. [CrossRef] 
7. Kremnizer, K.; Szczesny, M. Feynman graphs, rooted trees, and Ringel-Hall algebras. Commun. Math. Phys. 2009, 289, 561-577. [CrossRef]

8. Moser, J. Three integrable Hamiltonian systems connected with isospectral deformations. Adv. Math. 1975, 16, 197-220. [CrossRef]

9. Moser, J. Integrable Hamiltonian Systems and Spectral Theory; Edizioni della Normale, Publ. Scuola Normale Superiore; Springer: Berlin/Heidelberg, Germany, 1983.

10. Moser, J.; Zehnder, E. Notes on Dynamical Systems; Courant Lecture Notes in Math; American Mathematical Society, Courant Institute of Mathematical Sciences: New York, NY, USA, 2005.

11. Blackmore, D.; Prykarpatsky, A.K.; Samoylenko, V.H. Nonlinear Dynamical Systems of Mathematical Physics; World Scientific Publisher: Singapore, 2011.

12. Blackmore, D.; Prykarpatsky, Y.A.; Bogolubov, N.N.; Prykarpatski, A.K. Integrability of and differentialalgebraic structures for spatially 1D hydrodynamical systems of Riemann type. Chaos Solit. Fractals 2014, 59, 59-81. [CrossRef]

13. Blaszak, M. Bi-Hamiltonian Dynamical Systems; Springer: New York, NY, USA, 1998.

14. Faddeev, L.D.; Takhtadjan, L.A. Hamiltonian Methods in the Theory of Solitons; Springer: New York, NY, USA; Berlin, Germany, 1986.

15. Olver, P. Applications of Lie Groups to Differential Equations, 2nd ed.; Springer-Verlag: New York, NY, USA, 1993.

16. Dorfman, I. Dirac Structures and Integrability of Nonlinear Evolution Equations; Wiley: Chichester, UK, 1993.

17. Gel'fand, I.M.; Dorfman, I.Y. Hamiltonian operators and algebraic structures related to them (Russian). Funktsional. Anal. Prilozhen. 1979, 13, 13-30.

18. Dubrovin, B.A.; Novikov, S.P. Hamiltonian formalism of one-dimensional systems of hydrodynamic type and the Bogolyubov-Whitham averaging method. Sov. Math. Dokl. 1983, 27, 665-669.

19. Dubrovin, B.A.; Novikov, S.P. On Poisson brackets of hydrodynamic type. Sov. Math. Dokl. 1984, 30, 651-654.

20. Balinsky, A.A.; Novikov, S.P. Poisson brackets of hydrodynamic type, Frobenius algebras and Lie algebras. Sov. Math. Dokl. 1985, 32, 228-231.

21. Artemovych, O.D.; Blackmore, D.; Prykarpatski, A.K. Poisson brackets, Novikov-Leibniz structures and integrable Riemann hydrodynamic systems. J. Nonlinear Math. Phys. 2017, 24, 41-72. [CrossRef]

22. Prykarpatsky, A.K.; Artemovych, O.D.; Popowicz, Z.; Pavlov, M.V. Differential-algebraic integrability analysis of the generalized Riemann type and Korteweg-de Vries hydrodynamical equations. J. Phys. A Math. Theory 2010, 43, 295205. [CrossRef]

23. Prykarpatsky, Y.A.; Artemovych, O.D.; Pavlov, M.V.; Pryparpatsky, A.K. Diffrential-algebraic and bi-Hamiltonian integrability analysis of the Riemann hierarchy revisited. J. Math. Phys. 2012, 53, 103521. [CrossRef]

24. Bai, C.; Meng, D. Addendum: invariant bilinear forms. J. Phys. A 2001, 34, 8193-8197. [CrossRef]

25. Bai, C.; Meng, D. Transitive Novikov algebras on four-dimensional nilpotent Lie algebras. Int. J. Theoret. Phys. 2001, 40, 1761-1768. [CrossRef]

26. Bai, C.; Meng, D. The classification of Novikov algebras in low dimensions. J. Phys. A 2001, 34, $1581-1594$. [CrossRef]

27. Bai, C.; Meng, D. On fermionic Novikov algebras. J. Phys. A Math. Gen. 2002, 35, 10053-10063. [CrossRef]

28. Bai, C.; Meng, D.; He, L. On the Novikov algebra structures adapted to the automorphism structure in a Lie group. J. Geom. Phys. 2003, 45-2, 105-115. [CrossRef]

29. Osborn, J.M. Novikov algebras, Nova J. Algebra Geom. 1992, 1, 1-13.

30. Strachan, I.A.B.; Szablikowski, B.M. Novikov Algebras and a classification of multicomponent CamassaHolm equations. Stud. Appl. Math. 2014, 133, 84-117. [CrossRef]

31. Holm, D.D.; Ivanov, R.I. Multi-component generalizations of the $\mathrm{CH}$ equation: geometrical aspects, peakons and numerical examples. J. Phys. A 2010, 43, 492001. [CrossRef]

32. Bogdanov, L.V. Interpolating differential reductions of multidimensional integrable hierarchies. TMF 2011, 167, 354-363. [CrossRef]

33. Bogdanov, L.V.; Dryuma, V.S.; Manakov, S.V. Dunajski generalization of the second heavenly equation: Dressing method and the hierarchy. J. Phys. A Math. Theory 2007, 40, 14383-14393. [CrossRef]

34. Bogdanov, L.V.; Konopelchenko, B.G. On the heavenly equation and its reductions. J. Phys. A Math. Gen. 2006, 39, 11793-11802. [CrossRef] 
35. Doubrov, B.; Ferapontov, E.V. On the integrability of symplectic Monge-Ampère equations. J. Geom. Phys. 2010, 60, 1604-1616. [CrossRef]

36. Dunajski, M. Anti-self-dual four-manifolds with a parallel real spinor. R. Soc. Lond. Proc. Ser. A Math. Phys. Eng. Sci. 2002, 458, 1205-1222. [CrossRef]

37. Konopelchenko, B.G. Grassmanians $\operatorname{Gr}(N-1, N+1)$, closed differential $N-1$ forms and $N$-dimensional integrable systems. arXiv 2013, arXiv:1208.6129v2.

38. Krasil'shchik, I. A natural geometric construction underlying a class of Lax pairs. Lobachevskii J. Math. 2016, 37, 61-66. [CrossRef]

39. Sergyeyev, A. A simple construction of recursion operators for multidimensional dispersionless integrable systems. J. Math. Anal. Appl. 2017, 454, 468-480. [CrossRef]

40. Takasaki, K.; Takebe, T. SDiff(2) Toda equation-Hierarchy, Tau function, and symmetries. Lett. Math. Phys. 1991, 23, 205-214. [CrossRef]

41. Takasaki, K.; Takebe, T. Integrable hierarchies and dispersionless limit. Rev. Math. Phys. 1995, 7, $743-808$. [CrossRef]

42. Zakharevich, I. Nonlinear wave equation, nonlinear Riemann problem, and the twistor transform of Veronese webs. arXiv 2000, arXiv:math-ph/0006001.

43. Hentosh, O.; Prykarpatsky, Y.A.; Blackmore, D.; Prykarpatski, A.K. Lie-algebraic structure of Lax-Sato integrable heavenly equations and the Lagrange-d'Alembert principle. J. Geom. Phys. 2017, 120, $208-227$. [CrossRef]

44. Sergyeyev, A. New integrable (3+1)-dimensional systems and contact geometry. arXiv 2017, arXiv:1401.2122v4.

45. Schief, W.K. Self-dual Einstein spaces via a permutability theorem for the Tzitzeica equation. Phys. Lett. A 1996, 223, 55-62. [CrossRef]

46. Schief, W.K. Self-dual Einstein spaces and a discrete Tzitzeica equation. A permutability theorem link. In Symmetries And Integrability of Difference Equations; London Mathematical Society, Lecture Note Series 255; Clarkson, P., Nijhoff, F., Eds.; Cambridge University Press: Cambridge, UK, 1999; pp. 137-148.

47. Sergyeyev, A.; Szablikowski, B.M. Central extensions of cotangent universal hierarchy: $(2+1)$-dimensional bi-Hamiltonian systems. Phys. Lett. A 2008, 372, 7016-7023. [CrossRef]

48. Szablikowski, B. Hierarchies of Manakov-Santini Type by Means of Rota-Baxter and Other Identities. SIGMA 2016, 12, 022. [CrossRef]

49. Blackmore, D.; Hentosh, O.E.; Prykarpatski, A. The Novel Lie-Algebraic Approach to Studying Integrable Heavenly Type Multi-Dimensional Dynamical Systems. J. Gener. Lie Theory Appl. 2017, 11. [CrossRef]

50. Prykarpatski, A.K.; Hentosh, O.E.; Prykarpatsky, Y.A. Geometric Structure of the Classical Lagranged'Alambert Principle and its Application to Integrable Nonlinear Dynamical Systems. Mathematics 2017, 5, 75. [CrossRef]

51. Błaszak, M.; Szum, A. Lie algebraic approach to the construction of $(2+1)$-dimensional lattice-field and field integrable Hamiltonian equations. J. Math. Phys. 2001, 35, 4088-4116. [CrossRef]

52. Błaszak, M.; Szum, A.; Prykarpatsky, A. Central extension approach to integrable field and lattice-field systems in (2+1)-dimensions. Rep. Math. Phys. 1999, 37, 27-32. [CrossRef]

53. Cieśliński, J.; Prykarpatski, A.K. Discrete approximations on functional classes for the integrable nonlinear Schrödinger dynamical system: A symplectic finite-dimensional reduction approach. J. Math. Anal. Appl. 2015, 430, 279-295. [CrossRef]

54. Cieśliński, J.; Prykarpatski, A.K. The discrete nonlinear Schrödinger type hierarchy, its finite-dimensional reduction analysis and numerical integrability scheme. Nonlinear Oscillations 2017, 20, 228-265.

55. Benoist, Y. Une nilvariete non-affine. J. Differ. Geom. 1995, 41, 21-52. [CrossRef]

56. Burde, D. Affine structures on nilmanifolds. Int. J. Math. 1996, 7, 599-616. [CrossRef]

57. Kim, H. Complete left-invariant affine structures on nilpotent Lie groups. J. Differ. Geom. 1986, $24,373-394$. [CrossRef]

58. Perea, A.M. Flat left-invariant connections adapted to the automorphism structure of a Lie group. J. Differ. Geom. 1981, 16, 445-474. [CrossRef]

59. Zelmanov, E. A class of local translation-invariant Lie algebras (Russian). Dokl. Akad. Nauk SSSR 1987, 292, 1294-1297.

60. Semenov-Tian-Shansky, M. What is a classical R-matrix? Func. Anal. Appl. 1983, 17, 259-272. [CrossRef] 
61. Abraham, R.; Marsden, J.E. Foundations of Mechanics; Benjamin/Cummings Publisher: San Francisco, CA, USA, 1978.

62. Arnold, V.I. Mathematical Methods of Classical Mechanics; Springer: New York, NY, USA, 1989.

63. Oevel, W. Dirac constraints in field theory: Lifts of Hamiltonian systems to the cotangent bundle. J. Math. Phys. 1988, 29, 210-219. [CrossRef]

64. Oevel, W. R-structures, Yang-Baxter equations and related involution theorems. J. Math. Phys. 1989, 30, 1140-1149. [CrossRef]

65. Reyman, A.G.; Semenov-Tian-Shansky, M.A. Integrable Systems (Russian); The Computer Research Institute Publ.: Moscow, Russia; Izhvek, Russia, 2003.

66. Li, L.-C.; Parmentier, S. Nonlinear Poisson structures and r-matrices. Commun. Math. Phys. 1989, 125, 545-563. [CrossRef]

67. Godbillon, C. Geometrie Differentielle Et Mecanique Analytique; Hermann Publ.: Paris, France, 1969.

68. Prykarpatsky, A.K.; Mykytyuk, I.V. Algebraic Integrability of Nonlinear Dynamical Systems on Manifolds: Classical and Quantum Aspects; Kluwer Academic Publishers: Dordrecht, The Netherlands, 1998.

69. Bĺaszak, M.B.; Szablikowski, B.M. Classical R-matrix theory for bi-Hamiltonian field systems. J. Phys. A Math. Theory 2009, 42, 404002. [CrossRef]

70. Auslander, L. Simply transitive groups of affine motions. Am. Math. J. 1977, 99, 215-222. [CrossRef]

71. Osborn, J.M. Simple Novikov algebras with an idempotent. Commun. Algebra 1992, 20, 2729-2753.

72. Osborn, J.M.; Zelmanov, E. Nonassociative algebras related to Hamiltonian operarors in the formal calculus of variations. J. Pure Appl. Algebra 1995, 101, 335-352. [CrossRef]

73. Xu, X. On simple Novikov algebras and their irreducible modules Novikov algebras. J. Algebra 1996, 185, 905-934. [CrossRef]

74. Burde, D.; Dekimpe, K. Novikov structures on solvable Lie algebras. J. Geom. Phys. 2006, 56, $1837-1855$. [CrossRef]

75. Burde, D.; Dekimpe, K.; Vercammen, K. Novikov algebras and Novikov structures on Lie algebras. Linear Algebra Appl. 2008, 429, 31-41. [CrossRef]

76. Burde, D.; de Graaf, W. Classification of Novikov algebras. Appl. Algebra Eng. Commun. Comput. 2013, 24, 1-15. [CrossRef]

77. Baehr, H.C.; Dimakis, A.; Müller-Hoissen, F. Differential calculi on commutative algebras. J. Phys. A 1995, 28, 31973222. [CrossRef]

78. Burde, D. Simple left-symmetric algebras with solvable Lie algebras. Manuscripta Math. 1998, 95, $397-411$.

79. Jacobson, N. A note on nonassociative algebras. Duke Math. J. 1937, 3, 544-548. [CrossRef]

80. Farrand, S.M.; Finston, D.R. On the multiplication ideal of a nonassociative algebra. Arch. Math. 1987, 48, 298-818. [CrossRef]

81. Finston, D.R. On multiplicative algebras. Trans. Am. Math. Soc. 1986, 293, 807-818. [CrossRef]

82. Filippov, V.T. On right-symmetric and Novikon nil algebras of bounded index (Russian). Mat. Zametki 2001, 70, 289-295.

83. Jacobson, N. Lie Algebras; Interscience: New York, NY, USA, 1962.

84. Jacobson, N. Structure and Representations of Jordan Algebras; American Mathematical Society Colloquium Publications V. 39; AMS: Providence, RI, USA, 1968.

85. Zhevlakov, K.A.; Slinko, A.M.; Shestakov, I.P.; Shirshov, A.I. Rings That Are Nearly Associative, Translated From Russian By Harry F. Smoth; Academic Press: New York, NY, USA, 1982; pp. 4729-4738.

86. Levitzki, J.; On problem of A. Kurosh. Bull. Am. Math. Soc. 1946, 50, 1033-1035. [CrossRef]

(c) 2018 by the authors. Licensee MDPI, Basel, Switzerland. This article is an open access article distributed under the terms and conditions of the Creative Commons Attribution (CC BY) license (http:/ / creativecommons.org/licenses/by/4.0/). 